This article has been accepted for publication in Monthly Notices of the Royal Astronomical Society (C: 2020 The Authors Published by Oxford University Press on behalf of the Royal Astronomical Society. All rights reserved. 


\title{
New constraints on the magnetization of the cosmic web using LOFAR Faraday rotation observations
}

\author{
S. P. O’Sullivan ${ }^{\odot},{ }^{1,2 \star}$ M. Brüggen,${ }^{1}$ F. Vazza ${ }^{\odot},{ }^{1,3,4}$ E. Carretti ${ }^{\oplus},{ }^{4}$ N. T. Locatelli, ${ }^{3,4}$ \\ C. Stuardi ${ }^{\oplus},{ }^{3,4}$ V. Vacca, ${ }^{5}$ T. Vernstrom, ${ }^{6}$ G. Heald ${ }^{\oplus},{ }^{6}$ C. Horellou, ${ }^{7}$ T. W. Shimwell, ${ }^{8,9}$ \\ M. J. Hardcastle ${ }^{\odot}{ }^{10}$ C. Tasse $^{11}$ and H. Röttgering 9 \\ ${ }^{1}$ Hamburger Sternwarte, Universität Hamburg, Gojenbergsweg 112, Hamburg D-21029, Germany \\ ${ }^{2}$ School of Physical Sciences and Centre for Astrophysics \& Relativity, Dublin City University, Glasnevin D09 W6Y4, Ireland \\ ${ }^{3}$ Dipartimento di Fisica e Astronomia, Universitá di Bologna, Via Gobetti 92/3, I-40121 Bologna, Italy \\ ${ }^{4}$ Istituto di Radio Astronomia, INAF, Via Gobetti 101, I-40121 Bologna, Italy \\ ${ }^{5}$ INAF - Osservatorio Astronomico di Cagliari, Via della Scienza 5, I-09047 Selargius (CA), Italy \\ ${ }^{6}$ CSIRO Astronomy and Space Science, PO Box 1130, Bentley, WA 6102, Australia \\ ${ }^{7}$ Department of Space, Earth and Environment, Chalmers University of Technology, Onsala Space Observatory, SE-43992 Onsala, Sweden \\ ${ }^{8}$ ASTRON, the Netherlands Institute for Radio Astronomy, Postbus 2, NL-7990 AA Dwingeloo, the Netherlands \\ ${ }^{9}$ Leiden Observatory, Leiden University, PO Box 9513, NL-2300 RA Leiden, the Netherlands \\ ${ }^{10}$ Centre for Astrophysics Research, School of Physics, Astronomy and Mathematics, University of Hertfordshire, College Lane, Hatfield AL10 9AB, UK \\ ${ }^{11}$ GEPI \& USN, Observatoire de Paris, Université PSL, CNRS, 5 Place Jules Janssen, F-92190 Meudon, France
}

Accepted 2020 May 13. Received 2020 May 13; in original form 2020 February 7

\begin{abstract}
Measuring the properties of extragalactic magnetic fields through the effect of Faraday rotation provides a means to understand the origin and evolution of cosmic magnetism. Here, we use data from the LOFAR Two-Metre Sky Survey (LoTSS) to calculate the Faraday rotation measure (RM) of close pairs of extragalactic radio sources. By considering the RM difference $(\triangle \mathrm{RM})$ between physical pairs (e.g. double-lobed radio galaxies) and non-physical pairs (i.e. close projected sources on the sky), we statistically isolate the contribution of extragalactic magnetic fields to $\triangle \mathrm{RM}$ along the line of sight between non-physical pairs. From our analysis, we find no significant difference between the $\Delta \mathrm{RM}$ distributions of the physical and nonphysical pairs, limiting the excess Faraday rotation contribution to $<1.9 \mathrm{rad} \mathrm{m}^{-2}(\sim 95$ per cent confidence). We use this limit with a simple model of an inhomogeneous universe to place an upper limit of $4 \mathrm{nG}$ on the cosmological co-moving magnetic field strength on Mpc scales. We also compare the RM data with a more realistic suite of cosmological magnetohydrodynamical simulations that explore different magnetogenesis scenarios. Both magnetization of the largescale structure by astrophysical processes such as galactic and AGN outflows, and simple primordial scenarios with seed magnetic field strengths $<0.5 \mathrm{nG}$ cannot be rejected by the current data; while stronger primordial fields or models with dynamo amplification in filaments are disfavoured.
\end{abstract}

Key words: techniques: polarimetric-galaxies: active-galaxies: magnetic fields-largescale structure of Universe - radio continuum: galaxies.

\section{INTRODUCTION}

Uncovering the origin and understanding the evolution of cosmic magnetic fields is one of the key science goals for present and future radio telescopes (e.g. Gaensler, Beck \& Feretti 2004; Akahori et al. 2018). In addition to understanding the influence of magnetic fields on a range of different astrophysical scales, these studies can provide important information on the physics of the early Universe (Widrow et al. 2012; Jedamzik \& Pogosian 2020). In particular, detecting the presence of magnetic fields in cosmic filaments and voids can provide key constraints on magnetogenesis scenarios (Durrer \& Neronov 2013; Subramanian 2016), mainly because they are not as strongly modified as the magnetic fields in galaxies and galaxy cluster environments. Direct detection of the non-thermal synchrotron emission associated with fields in cosmic filaments may be possible (Vacca et al. 2018; Vazza et al. 2019), while an alternative approach is to use the Faraday rotation of linearly 
polarized radio sources to measure the field strength in thermal magnetized plasma along the line of sight (Kronberg \& Perry 1982; Oren \& Wolfe 1995; Kolatt 1998; Stasyszyn et al. 2010; Akahori, Gaensler \& Ryu 2014). This approach should also be possible in future large surveys of fast radio bursts (FRB), provided that thousands of FRB rotation measures (RMs) will be available (e.g. Hackstein et al. 2019).

The magnetic field properties of galaxies and the intergalactic medium in groups and clusters of galaxies are well studied (e.g. Carilli \& Taylor 2002; Laing et al. 2008; Beck 2015; van Weeren et al. 2019). However, the magnetic field properties of the pristine gas far outside galaxy clusters in filaments and voids are poorly constrained, with upper limits ranging from tens of nano-gauss (Ravi et al. 2016; Vernstrom et al. 2019) and less (Blasi, Burles \& Olinto 1999; Hackstein et al. 2016; Pshirkov, Tinyakov \& Urban 2016; Planck Collaboration XIX 2016b; Bray \& Scaife 2018) to lower limits of $\sim 10^{-17} \mathrm{G}$ (Neronov \& Vovk 2010; Dermer et al. 2011; Dolag et al. 2011; Tavecchio et al. 2011; Taylor, Vovk \& Neronov 2011). Improving our understanding of the strength and morphology of these fields will help to discriminate between competing models for the origin of cosmic magnetism, such as a primordial origin (Grasso \& Rubinstein 2001; Widrow 2002; Kulsrud \& Zweibel 2008) or at later times through AGN and/or galactic outflows (Zweibel \& Heiles 1997; Furlanetto \& Loeb 2001; Widrow 2002; Beck et al. 2013). Most notably, the fall off in field strength with distance from dense regions of the Universe is less steep in the case of a primordial origin compared to a later 'magnetic pollution' through outflows (Donnert et al. 2009; Vazza et al. 2017).

Constraining the magnetization of the Universe on large scales can also help test models of dark matter. For example, axion-like particles (ALPs) are a promising dark matter candidate (Raffelt \& Stodolsky 1988; Csáki et al. 2003), which are predicted to oscillate into high-energy photons (and back) in the presence of background magnetic fields (Horns et al. 2012). Photon-ALP oscillations are estimated to be possible on scales of a few Mpc in the presence of magnetic field strengths ranging from $\sim 1$ to $10 \mathrm{nG}$ (Montanino et al. 2017).

The focus of this paper is on using the Faraday RM of a sample of extragalactic radio sources to constrain the properties of the intergalactic magnetic field (IGMF) on large scales. This approach probes the thermal gas density-weighted field strength along the line of sight, where

$\left.\mathrm{RM}_{(\mathrm{rad} \mathrm{m}}^{-2}\right)=0.812 \int_{\text {source }}^{\text {telescope }} n_{e\left(\mathrm{~cm}^{-3}\right)} B_{\|(\mu \mathrm{G})} \mathrm{d} l_{(\mathrm{pc})}$,

with $B_{\|}$representing the line-of-sight magnetic field strength, $n_{e}$ the free electron number density, and $l$ the path-length through the magnetoionic medium. This is complementary to other radio studies that attempt to detect the faint synchrotron emission from relativistic electrons in the cosmic web between clusters of galaxies (e.g. Brown et al. 2017; Vernstrom et al. 2017; Botteon et al. 2018; Vacca et al. 2018; Govoni et al. 2019).

In order to assess the contribution of the IGMF to the RM, we need to study the contributions to the RM along the entire line of sight. For a statistical sample of background polarized radio sources, we are primarily concerned with the RM variance generated from extragalactic Faraday rotating material $\left(\sigma_{\mathrm{RM}, \mathrm{ex}}^{2}\right)$ that can be local or internal to the radio source itself or from the intergalactic medium on large scales. Furthermore, there is a large contribution from the interstellar medium (ISM) of the Milky Way $\left(\sigma_{\mathrm{RM}, \mathrm{MW}}^{2}\right)$, and a small contribution from the time-variable RM of the Earth's ionosphere $\left(\sigma_{\mathrm{RM}, \text { ion }}^{2}\right)$, in addition to measurement errors $\left(\sigma_{\mathrm{RM}, \mathrm{err}}^{2}\right)$. The total RM variance is then

$\sigma_{\mathrm{RM}}^{2}=\sigma_{\mathrm{RM}, \mathrm{ex}}^{2}+\sigma_{\mathrm{RM}, \mathrm{MW}}^{2}+\sigma_{\mathrm{RM}, \mathrm{ion}}^{2}+\sigma_{\mathrm{RM}, \mathrm{err}}^{2}$.

The majority of recent investigations of RM variance have been conducted at $1.4 \mathrm{GHz}$, mainly due to the catalogue of $37543 \mathrm{RMs}$ produced from the NRAO VLA Sky Survey data (NVSS; Condon et al. 1998; Taylor, Stil \& Sunstrum 2009). Most investigations have used this catalogue to study the properties of the Milky Way (e.g. Harvey-Smith, Madsen \& Gaensler 2011; Stil, Taylor \& Sunstrum 2011; Oppermann et al. 2012; Purcell et al. 2015; Hutschenreuter \& Enßlin 2019). However, Schnitzeler (2010) and Oppermann et al. (2015) modelled both the Galactic and extragalactic RM variance and found a best-fitting extragalactic RM variance of $\sim 7 \mathrm{rad} \mathrm{m}^{-2}$. Recently, Vernstrom et al. (2019) conducted an innovative study of close pairs of extragalactic RMs to isolate an extragalactic RM variance of $\sim 5-10 \mathrm{rad} \mathrm{m}^{-2}$. The $\mathrm{RM}$ variance local to radio sources has been well studied for individual objects, typically in groups or clusters of galaxies where the hot, magnetized intracluster gas can dominate the RM variance (e.g. Laing et al. 2008). However, the contribution of Faraday rotating material directly related to the radio sources themselves can be significant in some cases (e.g. Rudnick \& Blundell 2003; O'Sullivan et al. 2013; Anderson et al. 2018; Banfield et al. 2019; Knuettel et al. 2019). Importantly, since the RM variance local to radio sources can vary from tens to thousands of $\mathrm{rad} \mathrm{m}^{-2}$, isolating a population of low RM variance sources is a key objective for experiments that aim to probe foreground RM screens with high precision (Rudnick 2019). The ionospheric RM must also be considered (Sotomayor-Beltran et al. 2013) since the typical contribution is of $O\left(1 \mathrm{rad} \mathrm{m}^{-2}\right)$, which is similar to or larger than the signal from the IGMF that we want to probe.

In this paper, we present an RM study in quite a different regime for Faraday rotation, using the Low Frequency Array (LOFAR; van Haarlem et al. 2013) at $144 \mathrm{MHz}$. In particular, we use data from the ongoing LOFAR Two-Metre Sky Survey (LoTSS; Shimwell et al. 2019) from 120 to $168 \mathrm{MHz}$. This provides a wavelengthsquared coverage more than 600 times that of the NVSS. Since the accuracy with which one can measure Faraday rotation depends on the wavelength-squared coverage, the advantage of RM studies at $\mathrm{m}$-wavelengths is a dramatically higher precision on individual RM measurements (Neld et al. 2018; O'Sullivan et al. 2018b; Van Eck et al. 2018). However, the effect of Faraday depolarization increases substantially at long wavelengths, and while this provides important information on the properties of the magnetic field, it also means that a smaller fraction of radio sources are polarized at a level that can be detected (e.g. Farnsworth, Rudnick \& Brown 2011). This means that to reach a comparable polarized source density on the sky, observations at metre wavelengths need to be much deeper than cm-wavelengths (O'Sullivan et al. 2018a). To date, the majority of polarized sources at $\mathrm{m}$-wavelengths have been large FRII radio galaxies (e.g. Van Eck et al. 2018), whose polarized hotspots and/or outer lobe regions extend well beyond the host galaxy environment, making them excellent probes of the IGMF and ideal for this project.

In studying the extragalactic RM with these data, we follow the strategy of Vernstrom et al. (2019), hereafter V19, of taking the RM difference between close pairs ( $<20$ arcmin) and then separating the samples into physical and non-physical (or random) pairs. The physical pairs (PPs) are typically double-lobed radio galaxies, while the non-physical pairs are sources that are close in projection on the sky but physically located at different redshifts. The key insight upon which this experiment is based is that the RM variance due to the Milky Way should vary with pair angular separation in a similar manner for the physical and non-physical pairs, while 
the extragalactic RM variance due to the IGMF is expected to be larger for the non-physical pairs because of the much larger cosmic separation along the line of sight.

In Section 2, we describe the observational data and our classification scheme. The observational results are presented in Section 3. Two approaches to placing model limits on IGMFs are described in Section 4, while a discussion of the implications are given Section 5, followed by the conclusions in Section 6. Throughout this paper, we assume a $\Lambda \mathrm{CDM}$ cosmology with $\mathrm{H}_{0}=67.8 \mathrm{~km} \mathrm{~s}^{-1} \mathrm{Mpc}^{-1}$, $\Omega_{\mathrm{M}}=0.308$, and $\Omega_{\Lambda}=0.692$ (Planck Collaboration XIII 2016a).

\section{DATA}

The LoTSS is an ongoing survey of the northern sky with the LOFAR High Band Antennas, producing total intensity images and catalogues with an angular resolution of $\sim 6 \operatorname{arcsec}$ at $144 \mathrm{MHz}$ (Shimwell et al. 2019). From the second data release (DR2) survey pipeline (Tasse et al., in preparation), polarization data products (Stokes $Q, U$, and $V$ ) are also being produced at an angular resolution of 20 arcsec. In this work, we make use of the Stokes $Q$ and $U$ data to find linearly polarized radio sources and derive their Faraday RM values.

The polarization data used here has a frequency range of 120 $168 \mathrm{MHz}$ with a channel bandwidth of $97.6 \mathrm{kHz}$. In order to efficiently find linearly polarized radio sources, we employ the technique of RM synthesis (Burn 1966; Brentjens \& de Bruyn 2005) where one takes a Fourier transform of the complex linear polarization vector, $\boldsymbol{P}\left(\lambda^{2}\right)$, defined as

$\boldsymbol{P}\left(\lambda^{2}\right)=\int_{-\infty}^{\infty} \boldsymbol{F}(\phi) \mathrm{e}^{2 i \phi \lambda^{2}} \mathrm{~d} \phi$,

to obtain the Faraday dispersion function, $\boldsymbol{F}(\phi)$, which provides the distribution of polarized emission as a function of Faraday depth $(\phi)$ along the line of sight. In our case, the Faraday depth of the peak of $|\boldsymbol{F}(\phi)|$ is taken as the RM of the source. The LoTSS data provides an $\mathrm{RM}$ resolution of $\sim 1.15 \mathrm{rad} \mathrm{m}^{-2}$ with a maximum scale of $\sim 1.0 \mathrm{rad} \mathrm{m}^{-2}$ (i.e. no sensitivity to resolved Faraday depth structures), and a maximum $|\mathrm{RM}|$ of $\sim 170 \mathrm{rad} \mathrm{m}^{-2}$ (up to $\sim 450 \mathrm{rad} \mathrm{m}^{-2}$ with half the sensitivity). The time-variable absolute ionospheric RM correction was applied using RMEXTRACT ${ }^{1}$ as part of the standard PREFACTOR pipeline (e.g. de Gasperin et al. 2019). Residual ionospheric RM correction errors of $\sim 0.1-0.3 \mathrm{rad} \mathrm{m}^{-2}$ are expected across a single $8 \mathrm{~h}$ observation (Sotomayor-Beltran et al. 2013).

The polarization catalogue is produced by running RM synthesis ${ }^{2}$ on the Stokes $Q$ and $U$ images for each survey pointing out to a radius of $2^{\circ}$ from the pointing centre. The catalogue used here is produced from 268 survey pointings that, considering the large overlap between adjacent pointings, covers a sky area of $\sim 2000 \mathrm{deg}^{2}$. The pointings used were not from a single contiguous sky area but were chosen from DR2 pointings that had been processed up to 2019 May 1 . The pointings come from two (partially covered) areas of the sky, from RA of 7 to $19 \mathrm{~h}$ with Dec. from 25 to $70^{\circ}$, and $\mathrm{RA}$ of 23 to $3 \mathrm{~h}$ with Dec. from 10 to $40^{\circ}$. The large overlap between pointings means that the same polarized sources are often found in multiple pointings. These duplicate sources were identified and only the source closest to a pointing centre was retained. The Faraday depth range was limited to $\pm 120 \mathrm{rad} \mathrm{m}^{-2}$ with a sampling

\footnotetext{
${ }^{1}$ https://github.com/lofar-astron/RMextract

${ }^{2}$ https://github.com/mrbell/pyrmsynth
}

of $0.3 \mathrm{rad} \mathrm{m}^{-2}$, mainly due to computer processing and storage limitations. Searching over a larger Faraday depth range (with a finer frequency channelization) will be required to find sources in regions of the sky with high mean RM values, such as at low Galactic latitudes. A polarized source was catalogued if the peak in the Faraday dispersion function (FDF) was greater than 8 times the average noise in $Q$ and $U$ (i.e. $\sigma_{Q U}$, calculated from the rms of the wings of the real and imaginary parts of the FDF at $>100 \mathrm{rad} \mathrm{m}^{-2}$ ). For an $8 \sigma_{Q U}$ limit we expect a false detection rate of $\sim 10^{-4}$, compared to $5 \sigma_{O U}$ where it may be as high as $\sim 4$ per cent (George, Stil \& Keller 2012). The $Q$ and $U$ frequency spectra were extracted at the source location and RM synthesis ${ }^{3}$ was applied with a finer sampling of $0.1 \mathrm{rad} \mathrm{m}^{-2}$. The catalogued $\mathrm{RM}$ value was obtained from fitting a parabola to the amplitude of the complex FDF. The error in each RM value was calculated in the standard way (e.g. Brentjens \& de Bruyn 2005) as the RM resolution divided by twice the signal to noise (i.e. in our case this is $\sim 0.58 \sigma_{Q U} / P$ ), where $P$ is the peak polarized intensity in the FDF after correction for the polarization bias following George et al. (2012). Polarized sources in the Faraday depth range of -3 to $+1 \mathrm{rad} \mathrm{m}^{-2}$ were mainly discarded due to the presence of substantial contamination from instrumental polarization, which is shifted from $0 \mathrm{rad} \mathrm{m}^{-2}$ by the ionospheric RM correction. The focus on this work is to obtain an initial catalogue of close RM pairs. A more complete LOFAR RM catalogue is under construction with more uniform sky coverage, in addition to the inclusion of sources without a close RM pair (O'Sullivan et al., in preparation).

\subsection{Classification of RM pairs}

To obtain an initial sample of LOFAR RM pairs, we cross-matched the preliminary LOFAR RM catalogue $(\sim 1000$ candidate polarized sources over $\sim 2000 \mathrm{deg}^{2}$ ) with itself, including only pairs with separations $\leq 20$ arcmin. After removing self-matches, and limiting the minimum separation to 0.33 arcmin (i.e. the angular resolution of the data of $20 \mathrm{arcsec}$ ), in addition to further quality assurance checks, we were left with 349 pairs. This matches the approach of V19, except for the minimum separation, which was limited to $1.5 \mathrm{arcmin}$ in their study. All LOFAR pairs were restricted to come from the same pointing to avoid the systematic RM errors introduced by the different ionosphere corrections for different pointings. In fact, taking the RM difference between sources within the same pointing (as we describe later) removes the majority of the residual RM variance from the ionospheric RM correction, modulo directiondependent variations on scales $<20$ arcmin (de Gasperin et al. 2018), leaving mainly the measurement errors from the observational noise remaining. This means there is a more precise measurement of the $\mathrm{RM}$ difference between pairs compared to the individual uncertainty on any one RM measurement.

Visual inspection was used to separate sources into physical pairs (part of the same extragalactic radio source, e.g. two lobes) and non-physical, random pairs (RPs; i.e. physically unrelated sources projected close to each other on the sky). Classification of sources into physical and random pairs was done by S. P. O'Sullivan. This classification task was straightforward due to the high-fidelity LoTSS Stokes $I$ images available at both 20 and 6 arcsec resolution. All pairs are at Galactic latitude $|b|>20^{\circ}$, with no obvious clustering of physical or random pairs in particular parts of the sky. A Kolmogorov-Smirnov (KS) test provides no evidence for the

\footnotetext{
${ }^{3}$ https://github.com/CIRADA-Tools/RM
} 

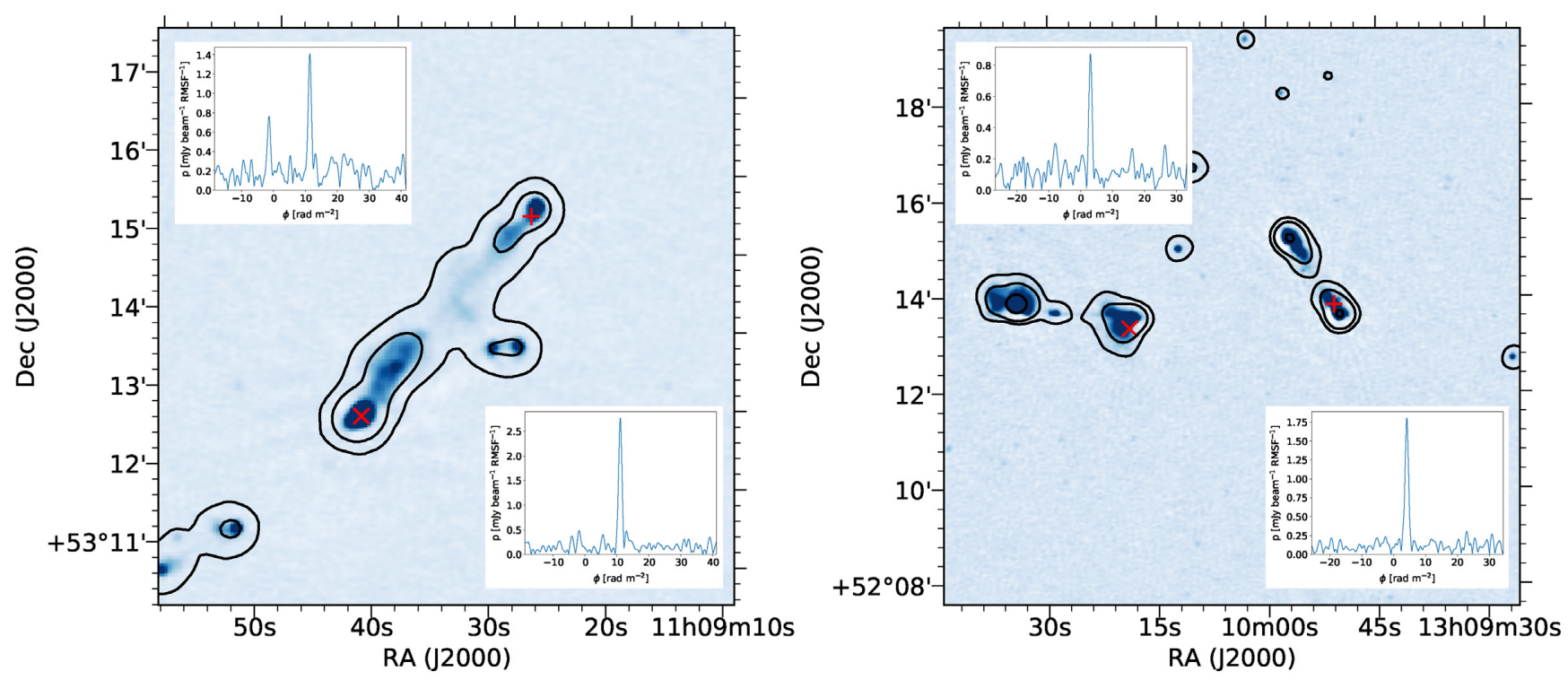

Figure 1. Example of a physical RM pair (left) and a non-physical, random RM pair (right). The random RM pair on the right is composed of two double-lobed radio galaxies, for which linear polarization is only detected in one lobe of each. The cross and plus symbols represent the location of the peak polarized intensity from which the RM value is taken. The contours outline the total intensity emission at an angular resolution of 20 arcsec, which is the same resolution as the corresponding polarization data. The colourscale shows the 6 arcsec total intensity emission, used for the classification into physical and random pairs. The insets show the Faraday spectra from the location of the cross and plus symbols in the top left and bottom right, respectively.

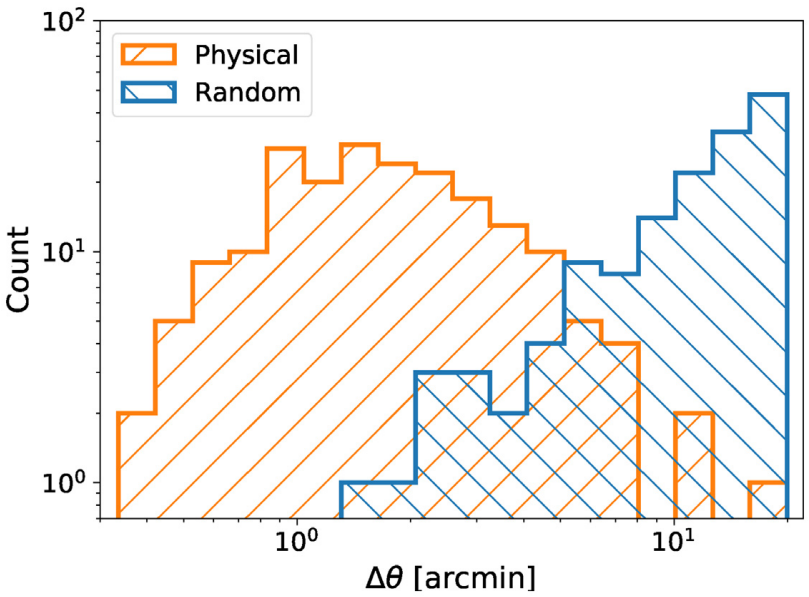

Figure 2. Histogram of the angular separation ( $\Delta \theta$, in units of arcminutes) of random (blue) and physical (orange) pairs. While the random pairs are typically found at larger angular separations, there is a significant overlap for random and physical pairs in the range of $\Delta \theta$ from 2 to $10 \mathrm{arcmin}$.

two samples having a different underlying distribution in Galactic latitude $(p=0.2)$. The highest number of pairs for a single catalogue source is 4 , with a median of 1 . Examples of physical and random pairs are shown in Fig. 1. Approximately 40 per cent of the random pairs have a compact source in the pair, while the resolved physical pairs are exclusively double-lobed radio galaxies.

\subsection{The $\mathbf{R M}$ difference in pairs}

We are interested in investigating the difference in $\mathrm{RM}(\Delta \mathrm{RM}=$ $\mathrm{RM}_{1}-\mathrm{RM}_{2}$ ) between pairs of sources (i.e. physical or random), in addition to the behaviour as a function of the angular separation $(\Delta \theta)$. Since the RM difference can be positive or negative, we expect a mean $\triangle \mathrm{RM}$ of zero for large samples. Therefore, we calculate the root mean square (rms) in $\Delta \mathrm{RM}$ as

$\Delta \mathrm{RM}_{\mathrm{rms}} \equiv \sqrt{\left\langle(\Delta \mathrm{RM})^{2}\right\rangle}=\sqrt{\frac{1}{N} \sum_{i}\left(\mathrm{RM}_{1}-\mathrm{RM}_{2}\right)_{i}^{2}}$.

The RM variance contributed by the measurement errors $\left(\sigma_{\mathrm{RM} \text {,err }}^{2}\right.$ in equation 2) can be subtracted from the total variance to analyse the astrophysical signal. We calculate this term from the quadrature sum of the errors on the individual RM measurements. Unless otherwise stated, the variance from measurement errors have been subtracted from the quoted $\Delta \mathrm{RM}_{\mathrm{rms}}$ values. For small samples or in the presence of outliers, the median can be a more robust statistic. Therefore, in our analysis we also consider the median of the absolute values of the RM difference (i.e. $|\Delta \mathrm{RM}|_{\text {median }}$ ).

\section{RESULTS}

In Fig. 2, we show the histogram of angular separations $(\Delta \theta)$ for our sample of 148 RPs and 201 PPs. In both cases, we limited the maximum angular separation to 20 arcmin, with the RPs extending down to $\sim 1.5$ arcmin and the PPs limited to the lower cut-off of $0.33 \mathrm{arcmin}$ (i.e. the angular resolution). The limit of $20 \mathrm{arcmin}$ was chosen because there are very few PPs above this separation. There is a clear difference in that the PPs are more often found at smaller angular separations (mean of 2 arcmin) than the random pairs (mean of 12 arcmin). This is expected since the PPs are limited to the linear size of the radio source, while the RPs have no such restriction. Since we want to compare the RPs and PPs, we are mainly interested in the region of significant overlap in angular separation between the two samples (in order to account for the Milky Way contribution). The overlap region we define here is from $\sim 2$ to $\sim 10$ arcmin, with 42 RPs and 75 PPs in this region (Table 1). For context, V19 found 317 PPs and 5111 RPs on angular scales from 1.5 to 20 arcmin. They chose an overlap region of 3-11 arcmin, which contained 158 RPs and 208 PPs. Although V19 had significantly more sources, our measurement errors are much lower, such that both experiments 
Table 1. Summary of results in the RM difference $(\Delta R M)$ between pairs.

\begin{tabular}{lrccc}
\hline Classification & $N$ & $\begin{array}{c}\Delta \mathrm{RM}_{\mathrm{rms}} \\
\left(\mathrm{rad} \mathrm{m}^{-2}\right)\end{array}$ & $\begin{array}{c}\Delta \mathrm{RM}_{\mathrm{rms} *} \\
\left(\mathrm{rad} \mathrm{m}^{-2}\right)\end{array}$ & $\begin{array}{c}|\Delta \mathrm{RM}|_{\text {median }} \\
\left(\mathrm{rad} \mathrm{m}^{-2}\right)\end{array}$ \\
\hline Random pairs (RP) & 148 & $6.0 \pm 0.5$ & $5.5 \pm 0.4$ & $1.5 \pm 1.4$ \\
Physical pairs (PP) & 201 & $1.4 \pm 0.1$ & $1.4 \pm 0.1$ & $0.7 \pm 0.6$ \\
RP: 2-10 arcmin & 42 & $5.1 \pm 0.8$ & $1.8 \pm 0.3$ & $1.2 \pm 0.6$ \\
PP: 2-10 arcmin & 75 & $1.4 \pm 0.2$ & $1.4 \pm 0.2$ & $0.9 \pm 0.6$
\end{tabular}

Note. $\Delta \mathrm{RM}_{\mathrm{rms} *}$ : Trimmed $\mathrm{rms}$, with one outlier from the random pairs removed.

Table 2. Table of the coordinates, angular separation and RM values of all sources in the sample. The ID column indicates classification as a random or physical pair with the ' $r$ ' or 'p' suffix. The nominal RM error value does not include the error from the ionosphere RM correction, and thus is only valid in the case of taking the difference in RM between pairs in this catalogue.

\begin{tabular}{|c|c|c|c|c|c|}
\hline ID & $\begin{array}{c}\text { RA } \\
(\mathrm{J} 2000)\end{array}$ & $\begin{array}{l}\text { Dec. } \\
(\mathrm{J} 2000)\end{array}$ & $\begin{array}{c}\Delta \theta \\
(\operatorname{arcmin})\end{array}$ & $\begin{array}{c}\mathrm{RM} \\
\left(\mathrm{rad} \mathrm{m}^{-2}\right)\end{array}$ & $\begin{array}{l}\mathrm{RM} \text { error } \\
\left(\mathrm{rad} \mathrm{m}^{-2}\right)\end{array}$ \\
\hline $1 p$ & 00:18:09.27 & 31:01:19.19 & 2.48 & -76.848 & 0.045 \\
\hline $1 p$ & 00:18:08.39 & $31: 03: 47.53$ & 2.48 & -77.338 & 0.016 \\
\hline $2 p$ & 00:29:00.04 & $29: 42: 15.88$ & 1.22 & -62.950 & 0.010 \\
\hline $2 p$ & 00:29:05.56 & $29: 42: 01.73$ & 1.22 & -60.584 & 0.007 \\
\hline $3 p$ & 00:44:34.09 & $12: 11: 26.59$ & 0.80 & -15.581 & 0.013 \\
\hline $3 p$ & $00: 44: 36.24$ & $12: 10: 50.60$ & 0.80 & -14.323 & 0.005 \\
\hline $4 p$ & $00: 45: 59.20$ & $22: 26: 54.03$ & 7.50 & -45.683 & 0.016 \\
\hline $4 p$ & $00: 46: 31.67$ & $22: 27: 06.75$ & 7.50 & -48.699 & 0.026 \\
\hline $5 \mathrm{p}$ & $00: 46: 54.30$ & $12: 57: 06.82$ & 3.56 & -12.789 & 0.006 \\
\hline $5 p$ & $00: 46: 52.13$ & $12: 53: 35.36$ & 3.56 & -13.192 & 0.020 \\
\hline $6 r$ & $00: 47: 06.84$ & $12: 44: 52.99$ & 12.61 & -11.804 & 0.030 \\
\hline $6 r$ & $00: 46: 54.30$ & $12: 57: 06.82$ & 12.61 & -12.789 & 0.006 \\
\hline $7 p$ & 00:51:02.21 & $13: 13: 37.38$ & 5.52 & -14.196 & 0.054 \\
\hline $7 p$ & 00:50:44.09 & $13: 16: 56.84$ & 5.52 & -17.856 & 0.033 \\
\hline $8 \mathrm{r}$ & $00: 53: 23.20$ & $33: 27: 25.21$ & 8.87 & -57.024 & 0.016 \\
\hline $8 \mathrm{r}$ & 00:52:40.78 & $33: 26: 51.98$ & 8.87 & -63.782 & 0.039 \\
\hline $9 p$ & 01:01:23.40 & $29: 28: 52.50$ & 1.85 & -67.635 & 0.043 \\
\hline $9 p$ & 01:01:30.60 & $29: 27: 53.26$ & 1.85 & -70.170 & 0.028 \\
\hline
\end{tabular}

Note. Except of the full table that is available online.

have comparable statistical power. The individual RM values for each pair are provided in Table 2 .

Fig. 3 shows the individual values of $(\Delta \mathrm{RM})^{2}$ and $\Delta \theta$ for each source pair, with the RPs indicated by plus symbols and the PPs indicated by cross symbols. The mean RM error for our sample is $0.026 \mathrm{rad} \mathrm{m}^{-2}$, and has a small contribution to the overall variance. The variance added by the measurement errors (i.e. $\sigma_{\mathrm{RM} \text {,err }}^{2}$ in equation (2)) for physical and random pairs as a function of angular size is approximately constant, and shown in Fig. 3 as dashed and dot-dashed lines with values of $\sim 0.0018 \mathrm{rad}^{2} \mathrm{~m}^{-4}$.

The rms of the RM difference for all RPs, $\Delta \mathrm{RM}_{\mathrm{rms}, \mathrm{RP}}=$ $6.0 \pm 0.5 \mathrm{rad} \mathrm{m}^{-2}$ while $\Delta \mathrm{RM}_{\mathrm{rms}, \mathrm{PP}}=1.4 \pm 0.1 \mathrm{radm}^{-2}$ (in all cases we quote the rms with the error variance subtracted, and the associated uncertainty is the standard error in the mean). $\mathrm{KS}$ and Anderson-Darling (AD) tests indicate that the difference between RPs and PPs is significant at $>5 \sigma$ (with $p$-values of $\sim 10^{-7}$ and $\sim 10^{-4}$, respectively). The empirical cumulative distribution function $(\mathrm{ECDF})$ of $(\triangle \mathrm{RM})^{2}$ is shown in Fig. 4, with all PPs and RPs shown with dotted lines. This difference is dominated by the general trend of larger $\Delta \mathrm{RM}$ variations at larger angular separations (Fig. 3), as is expected if the Milky Way ISM is a significant contributing factor to the RM variance on these angular scales (e.g. Stil et al. 2011).

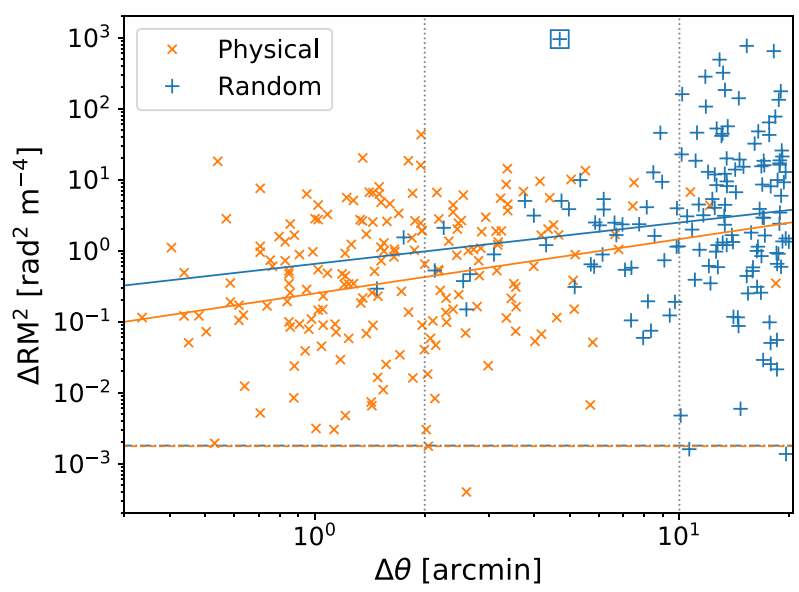

Figure 3. Scatter plot of the squared difference in $R M,(\Delta R M)^{2}$ in units of $\mathrm{rad}^{2} \mathrm{~m}^{-4}$, between pairs of radio sources versus the angular separation ( $\Delta \theta$, in units of arcminutes). Physical pairs are shown as orange cross symbols while the random pairs are shown as blue plus symbols (with the outlier highlighted by a box). Power-law fits to the physical and random pair data are shown as solid orange and blue lines, respectively. The small, constant contributions to $(\Delta \mathrm{RM})^{2}$ from the measurement errors are shown for physical and random pairs as horizontal orange dashed and blue dotdashed lines, respectively. The grey dotted vertical lines bound the overlap region of $2-10$ arcmin.

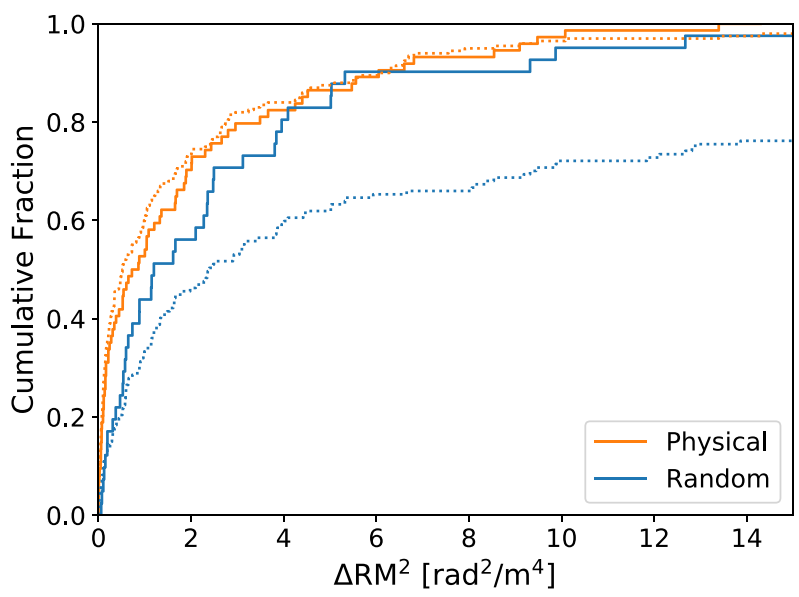

Figure 4. ECDFs of the squared difference in $R M,(\Delta R M)^{2}$ in units of $\mathrm{rad}^{2} \mathrm{~m}^{-4}$, between pairs of radio sources. The dashed blue and orange lines correspond to all the data for physical and random pairs, respectively, while the solid blue and orange lines show only the corresponding data for physical and random pairs in the overlapping region of angular separation from 2 to 10 arcmin.

If we restrict the comparison only to the region of significant overlap in angular separation (i.e. 2-10 $\operatorname{arcmin}$ ), then we have $\Delta \mathrm{RM}_{\mathrm{rms}, \mathrm{RP}}=5.1 \pm 0.8 \mathrm{radm}^{-2}$ and $\Delta \mathrm{RM}_{\mathrm{rms}, \mathrm{PP}}=$ $1.4 \pm 0.1 \mathrm{radm}^{-2}$. However, the rms for the RPs is strongly affected by one outlier, with the highest value of $\Delta \mathrm{RM}_{\mathrm{rms}}$ in the sample of $\sim 954 \mathrm{rad}^{2} \mathrm{~m}^{-4}$ (highlighted by a box in Fig 3). Therefore, we introduce the 'trimmed rms' $\left(\Delta \mathrm{RM}_{\mathrm{rms} *}\right)$ with this outlier removed. This reduces the rms of the RPs to $\Delta \mathrm{RM}_{\mathrm{rms}, \mathrm{RP}}=$ $1.8 \pm 0.3 \mathrm{rad} \mathrm{m}^{-2}$, giving a difference of $0.4 \pm 0.3 \mathrm{rad} \mathrm{m}^{-2}$ between the RPs and PPs between 2 and 10 arcmin. The difference in the median values of $|\Delta R M|$ for RPs and PPs in the overlap region 


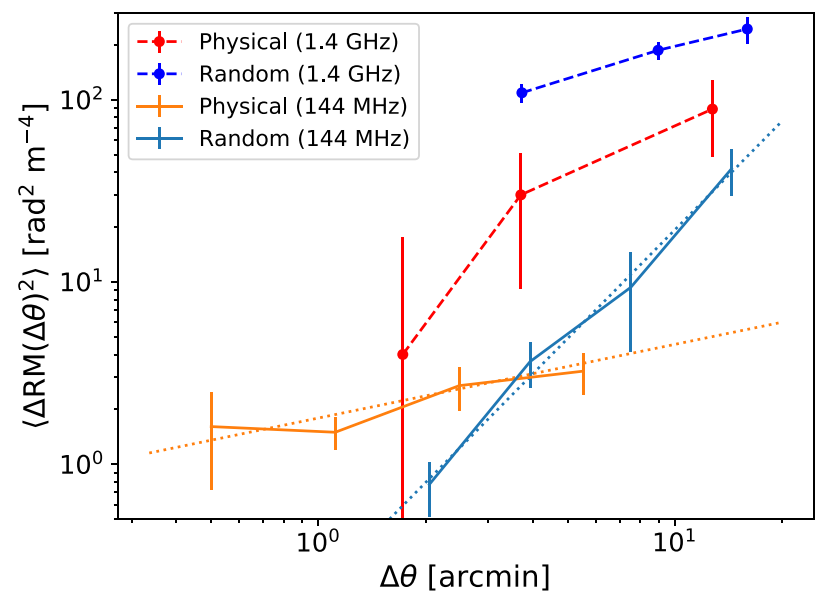

Figure 5. Plot of the RM structure functions [i.e. mean bins of $(\Delta \mathrm{RM})^{2}$ as a function of the pair angular separation, $\Delta \theta$ ] with the noise power from measurement errors subtracted, for PPs (orange) and RPs (blue) at $144 \mathrm{MHz}$. The orange and blue dotted lines show the power-law fits to the PPs and RPs, respectively. The RPs bin at the smallest angular separation has only seven data points, and may be unreliable. For comparison, we also include the structure function results from the $1.4 \mathrm{GHz}$ data of Vernstrom et al. (2019) for PPs (red circles) and RPs (dark blue circles), connected by dashed lines.

is $0.3 \pm 0.8 \mathrm{rad} \mathrm{m}^{-2}$. For the uncertainties in the median $|\Delta \mathrm{RM}|$, we use the median absolute deviation (i.e. half the interquartile range). These results are summarized in Table 1. The ECDFs of $(\Delta \mathrm{RM})^{2}$ for only those RPs and PPs in the overlapping angular separation region of 2-10 arcmin are shown with solid lines in Fig. 4. KS and AD tests indicate that the RPs and PPs in the overlap region are not significantly different for these sample sizes ( $p$-values of 0.17 and 0.06 , respectively). The exclusion of the outlier does not significantly affect the KS or AD test results ( $p$-values of 0.20 and 0.08). Therefore, based on the difference in the trimmed rms $\left(\Delta \mathrm{RM}_{\mathrm{rms} *}\right)$ values of $0.4 \pm 0.3 \mathrm{rad} \mathrm{m}^{-2}$ and the difference in the median values of $|\Delta R M|$ of $0.3 \pm 0.8 \mathrm{radm}^{-2}$, we consider a conservative upper limit on the excess Faraday rotation contribution between RPs to be $1.9 \mathrm{rad} \mathrm{m}^{-2}$ (i.e. the median difference plus twice the uncertainty). We use this upper limit to derive a model limit on extragalactic magnetic fields in Section 4.1.

\subsection{Trends in $\Delta R M$ as a function of angular separation}

We also fit a power-law function to the RPs and PPs data, $(\Delta \mathrm{RM})^{2}=$ $k \Delta \theta^{\gamma}$, where $k$ is a constant with units of $\operatorname{rad}^{2} \mathrm{~m}^{-4} \operatorname{arcmin}^{-\gamma}$. We find $k_{\mathrm{RP}}=0.6 \pm 1.0, \gamma_{\mathrm{RP}}=0.6 \pm 0.4$ and $k_{\mathrm{PP}}=0.25 \pm 0.04$, $\gamma_{\mathrm{PP}}=0.8 \pm 0.2$. These fits are shown in Fig. 3. The difference in $(\Delta \mathrm{RM})^{2}$ between the power-law fits at the average separation in the overlapping region (i.e. $6 \mathrm{arcmin}$ ) is $\sim 0.8 \mathrm{rad}^{2} \mathrm{~m}^{-4}$. Attempts at fitting only the data in the overlapping region were poorly constrained, so we do not include them here.

To more directly compare with the results of V19, we calculate the mean of $(\Delta R M)^{2}$ as a function of the angular separation, i.e. the $\mathrm{RM}$ structure function (SF), with

$\left\langle\Delta \mathrm{RM}(\Delta \theta)^{2}\right\rangle=\frac{1}{N} \sum_{i}\left[\mathrm{RM}_{1}(\theta)-\mathrm{RM}_{2}(\theta+\Delta \theta)\right]_{i}^{2}$.

This is done separately for the RPs and PPs, and they are plotted in Fig. 5 in addition to the V19 RM structure functions. By fitting power laws to these data in a similar manner to above, with $\left\langle(\Delta \mathrm{RM})^{2}\right\rangle=k_{\mathrm{SF}} \Delta \theta^{\gamma_{\mathrm{SF}}}$, we find $k_{\mathrm{SF}, \mathrm{RP}}=0.2 \pm 0.1, \gamma_{\mathrm{SF}, \mathrm{RP}}=$ $1.9 \pm 0.2$ (with the outlier removed) and $k_{\mathrm{SF}, \mathrm{PP}}=1.8 \pm 0.3, \gamma_{\mathrm{SF}, \mathrm{PP}}=$ $0.4 \pm 0.1$. These fits are shown by dotted lines in Fig. 5. The values of $\gamma_{\mathrm{SF}}$ are in stark contrast with those found in V19 $\left(\gamma_{\mathrm{SF}, \mathrm{RP}, \mathrm{NVSS}}=\right.$ $0.6 \pm 0.1, \gamma_{\mathrm{SF}, \mathrm{PP}, \mathrm{NVSS}}=0.8 \pm 0.2$ ) with the RPs slope being much steeper than in V19 and the PPs slope being much flatter. Also notable is that the overall amplitude is smaller in both cases compared to V19 $\left(k_{\mathrm{SF}, \mathrm{RP}, \mathrm{NVSS}}=50 \pm 30, k_{\mathrm{SF}, \mathrm{PP}, \mathrm{NVSS}}=11 \pm 15\right)$. These differences have important implications for the nature of the extragalactic Faraday rotating material and are addressed in the next section.

\subsection{Comparison with $\mathrm{RM}$ data at $1.4 \mathrm{GHz}$}

We find that 203 out of the 698 source components in this study ( $\sim 29$ per cent) have corresponding $\mathrm{RM}$ values at $1.4 \mathrm{GHz}$ in the Taylor et al. (2009) catalogue. The vast majority, 91 per cent (97 per cent), of the corresponding RM values are consistent within $3 \sigma(5 \sigma)$ of the combined RM error. The LOFAR sources that are not in the NVSS RM catalogue are on average $\sim 3$ times fainter in total intensity at $144 \mathrm{MHz}$. This means that the majority of the LOFAR polarized sources are too faint to be detected in the NVSS, while the majority of the NVSS sources are depolarized at LOFAR frequencies (e.g. O'Sullivan et al. 2018a). For those pairs that have counterparts in the V19 catalogue, we find $\Delta \mathrm{RM}_{\mathrm{rms}, \mathrm{RP}, \mathrm{NVSS}} \sim 18 \mathrm{rad} \mathrm{m}^{-2}$ and $\Delta \mathrm{RM}_{\mathrm{rms}, \mathrm{PP}, \mathrm{NVSS}} \sim 5 \mathrm{rad} \mathrm{m}^{-2}$, which is consistent with the results presented in V19. However, for the exact same sources we find $\Delta \mathrm{RM}_{\mathrm{rms}, \mathrm{RP}, \mathrm{LOFAR}} \sim 5 \mathrm{rad} \mathrm{m}^{-2}$ and $\Delta \mathrm{RM}_{\mathrm{rms}, \mathrm{PP}, \mathrm{LOFAR}} \sim 2 \mathrm{rad} \mathrm{m}^{-2}$. Furthermore, the median degrees of polarization for these RPs and PPs at $1.4 \mathrm{GHz}$ are $\sim 7$ per cent and $\sim 11$ per cent, respectively, while at $144 \mathrm{MHz}$ they are significantly lower at $\sim 1.5$ per cent and $\sim 3$ per cent, respectively.

This large difference in both the RM variance and degree of polarization of the same sources at $1.4 \mathrm{GHz}$ and $144 \mathrm{MHz}$ is most likely related to the broader range of Faraday depths that are probed local to the sources at $1.4 \mathrm{GHz}$. A plausible scenario is that the LOFAR observations are only sensitive to the low RM variance regions of these sources, and that the differences between the V19 results and those presented here are due to the RM properties of the local source environment. The difference in angular resolution between these studies (a factor of 3) may also play a role and a more detailed investigation is needed, including high angular resolution observations at $1.4 \mathrm{GHz}$.

The above hypothesis is supported by comparison of the RM structure functions at $144 \mathrm{MHz}$ and $1.4 \mathrm{GHz}$ (analysed in Section 3.1 and shown in Fig. 5). The difference between the RP data at $1.4 \mathrm{GHz}$ and $144 \mathrm{MHz}$ is $\sim 10 \mathrm{rad} \mathrm{m}^{-2}$, which is comparable to the total extragalactic RM variance estimated by V19, Schnitzeler (2010) and Oppermann et al. (2015). This likely reflects the typical contribution to the $\mathrm{RM}$ variance at $1.4 \mathrm{GHz}$ provided by the magnetized intragroup/intracluster medium surrounding radio galaxies. In this case, the Faraday depolarization caused by these environments is sufficient to depolarize the majority of sources below the detection threshold at $144 \mathrm{MHz}$, leaving only the low RM variance regions of some of these sources detectable with LOFAR.

The steeper slope of the RM structure function for RPs (compared to V19, see Fig. 5) may more cleanly reflect the RM variance from the Milky Way on these angular scales, if the extragalactic RM variance contribution is indeed much lower for the LOFAR data (more data for the PPs at large angular separations are needed to test this hypothesis). The flattening of the slope of the RM structure 


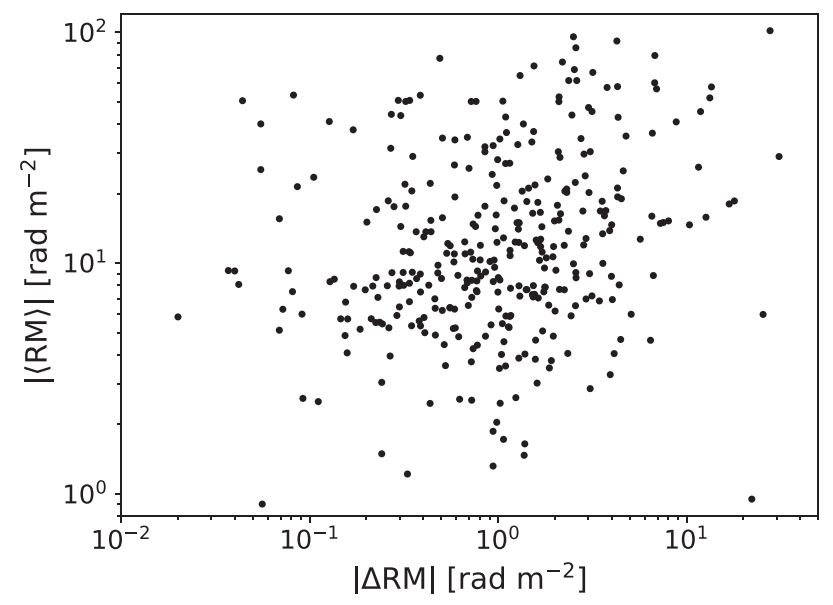

Figure 6. Plot of the average RM, $|\langle R M\rangle|$, versus the RM difference, $|\Delta \mathrm{RM}|$, for each pair. A Spearman rank test indicates they are weakly correlated, with a correlation coefficient of 0.2 , at a significant level ( $p$ value $\sim 10^{-5}$ ).

function towards smaller angular scales (as probed by the PPs), may reflect a growing contribution of the extragalactic $\mathrm{RM}$ variance (relative to the Milky Way), with $\left\langle(\Delta \mathrm{RM})^{2}\right\rangle \lesssim 1.6 \mathrm{rad}^{2} \mathrm{~m}^{-4}$ on the smallest angular separations (Fig. 5). We use this limit as a constraint for cosmological magnetohydrodynamical (MHD) simulations in Section 4.2.

If the Milky Way dominates the RM variance, then we might expect the average RM of each pair to be correlated with $\Delta R M$, because the average RM is known to be dominated by the Milky Way (e.g. Oppermann et al. 2012). In Fig. 6, we plot the absolute value of the average RM, $|\langle\mathrm{RM}\rangle|$, versus the absolute value of the $\mathrm{RM}$ difference, $|\Delta R M|$, for each pair. A Spearman rank test indicates these quantities are weakly correlated (correlation coefficient of $0.23)$ with a significance of $\sim 4.4 \sigma\left(p\right.$-value $\left.\sim 10^{-5}\right)$. The correlation for PPs is slightly stronger $\left(0.26, p\right.$-value $\left.\sim 10^{-4}\right)$ than for RPs $(0.20$, $p$-value $\left.\sim 10^{-2}\right)$. This indicates that, as expected, the Milky Way contributes to the RM variance even on these small angular scales. However, it remains unclear what the exact contribution is relative to the extragalactic RM variance. A much higher surface density of polarized sources on the sky is required to accurately estimate the Milky Way RM contribution for this data set

\section{SIMULATIONS}

\subsection{Monte Carlo modelling of extragalactic RM pairs}

In order to understand the implications for the properties of IGMFs based on the results in Section 3, we develop some basic simulations of extragalactic Faraday rotation. We use a model of an inhomogeneous evolving universe, with an initial cosmological magnetic field, the strength of which is scaled with the local density variations. This model allows us to calculate the RM along cosmic sightlines to PPs and RPs for different angular and redshift separations. We investigate a wide range of initial magnetic field strengths and correlation lengths, which are then constrained by the RM observations. In Section 4.1.1, we comment on some of the limitations of this model and compare this approach with simpler models in Section 4.1.2.

Following Blasi et al. (1999) and Pshirkov et al. (2016), we model the electron number density along cosmic lines of sight as $n_{e}(z)=$ $n_{e}(0)\left(1+\delta_{e}\right)(1+z)^{3}$, with $n_{e}(0)=1.8 \times 10^{-7} \mathrm{~cm}^{-3}$ and $\delta_{e}$ being the electron overdensity. We draw the electron overdensity $\delta_{e}$ from a lognormal distribution, with $\delta_{e}$ varying on scales of the Jeans length, $\lambda_{J}(z) \sim 2.3(1+z)^{-1.5} \mathrm{Mpc}$. The lognormal distribution is given by

$$
P\left(\delta_{e}\right)=\frac{1}{\sqrt{2 \pi} \sigma_{e}\left(1+\delta_{e}\right)} \exp \left\{-\frac{\left[\ln \left(1+\delta_{e}\right)-\mu_{e}(z)\right]^{2}}{2 \sigma_{e}^{2}}\right\},
$$

where the mean $\left(\mu_{e}\right)$ and standard deviation $\left(\sigma_{e}\right)$ of the logarithm of the $\delta_{e}$ distribution are constrained from observations of the Lyman $\alpha$ forest (e.g. Bi \& Davidsen 1997), with $\sigma_{e}(z)=0.08+5.37(1+$ $z)^{-1}-4.21(1+z)^{-2}+1.44(1+z)^{-3}$ and $\mu_{e}(z)=-\sigma_{e}(z)^{2} / 2$. For close pairs of sources we also need to include a prescription for the correlation of the densities along adjacent lines of sight. For this we use the two-point galaxy correlation function $\xi(r, z)=\left(r / r_{0}\right)^{-\gamma}(1$ $+z)^{-(2+\gamma)}$, including its expected redshift evolution, with $\gamma \sim 1.8$ and $r_{0} \sim 5 h^{-1}$ Mpc (e.g. Mo, van den Bosch \& White 2010). We consider this correlation function valid for scales between 0.2 and $30 \mathrm{~h}^{-1} \mathrm{Mpc}$. Practically, we implement the correlated draws using a bi-variate Gaussian distribution before taking the exponential, where the off-diagonal terms of the covariance matrix are given by $\xi_{G}=\ln (1+\xi)$ (e.g. Coles \& Jones 1991; Chuang et al. 2015; Baratta et al. 2019). For separations between adjacent cells larger than $30 \mathrm{~h}^{-1} \mathrm{Mpc}$, we draw from an uncorrelated lognormal distribution, while the same density is assigned for cell separations less than $0.2 h^{-1} \mathrm{Mpc}$.

We model the magnetic field strength as a scaled function of the density and redshift following $B(z)=B_{0}\left[n_{e}(z) / n_{e}(0)\right]^{2 / 3}$, which is a reasonable expectation in the case of isotropic gas compression (e.g. Locatelli, Vazza \& Domínguez-Fernández 2018). In this case, we have $B_{0}$ as the co-moving cosmological magnetic field strength in $\mathrm{nG}$. The correlation length of the magnetic field $\left(l_{B}\right)$ is set in fractions of the Jeans length, with random orientations assigned at each step by multiplying the amplitude of the field by a number drawn from a uniform $[-1,1]$ distribution. The RM values were obtained by summing the RM contributions over all cells along an individual line of sight (from the source to us), while accounting for the RM redshift dilution of $(1+z)^{2}$ in each cell.

Since we do not know the redshift distribution of our sample, we randomly draw sources from a lognormal redshift distribution $\left(\mu_{z}=-1, \sigma_{z}=1\right)$, which has a median of $z \sim 0.37$ and is consistent with the redshift distribution of polarized extragalactic radio sources (O'Sullivan et al. 2018a; Hardcastle et al. 2019; Vernstrom et al. 2019). However, we limit the redshift to a maximum of 1 for the PPs and 4 for the RPs following the corresponding maximum redshifts found in V19. We note that the actual redshift distribution of the LOFAR data may be somewhat different because the sources are typically fainter in total intensity that those at $1.4 \mathrm{GHz}$ (Section 3.2). Similarly, we do not know the projected linear size distribution of our physical pairs, but we can again reasonably model this as a lognormal distribution $\left(\mu_{\mathrm{ls}}=-1, \sigma_{\mathrm{ls}}=1\right)$ in Mpc units based on the projected linear size distributions for LOFAR radio galaxies in Hardcastle et al. (2019). For an angular size ranging from 2 to 10 arcmin, this gives a maximum linear size of $\sim 5 \mathrm{Mpc}$ and a minimum of $\sim 24 \mathrm{kpc}$, which is consistent with the range of linear sizes of LOFAR polarized sources (O'Sullivan et al. 2018a). In general, this aspect of the analysis can be substantially improved when the redshifts of the LOFAR polarized sources become available in the upcoming LoTSS DR2 value-added catalogue.

For the PPs, we first draw the redshift of the radio galaxy, then the linear size, and compute the separation, $\theta$, between the pair using 


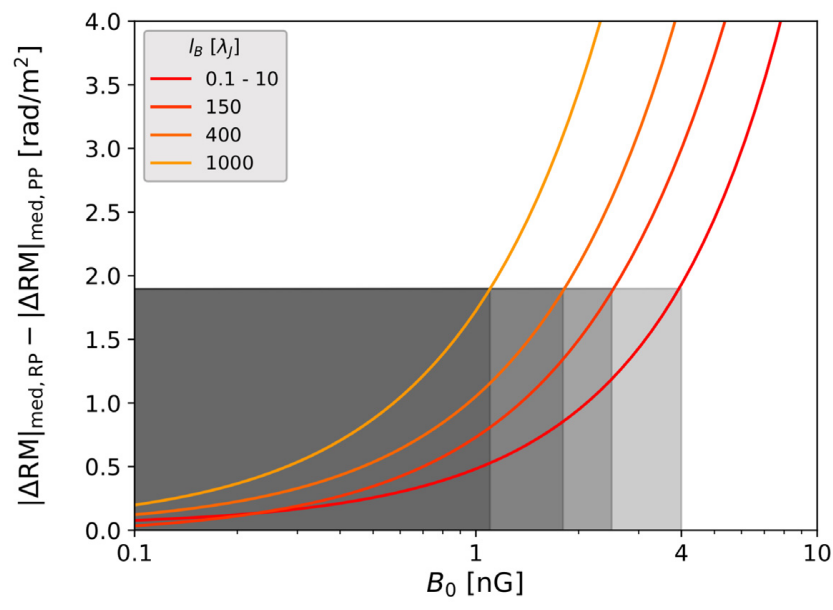

Figure 7. Plot of $|\Delta R M|_{\text {median, } R P}-|\Delta R M|_{\text {median,PP }}$ (in $\mathrm{rad} \mathrm{m}^{-2}$ ) versus the input initial cosmological magnetic field strength ( $B_{0}$ in $\mathrm{nG}$ ), provided by the inhomogeneous universe model, described in Section 4.1. The lines trace the growth in the difference in the Faraday rotation between RPs and PPs for increasing values of $B_{0}$. The shaded regions outline the upper limits on $B_{0}$ for various magnetic field correlation lengths $\left(l_{B}\right.$ in units of the Jeans length, $\lambda_{J}$ ), provided by the points at which the upper limit of $1.9 \mathrm{rad} \mathrm{m}^{-2}$ (derived in Section 3) intersects with the lines. The line furthest to the right defines the upper limit of $B_{0} \lesssim 4 \mathrm{nG}$ on Mpc scales.

the angular diameter distance. We construct two sightlines to the radio galaxy, only allowing the range $2<\theta<10$ arcmin, to obtain the RM for each sightline, before calculating the RM difference (in a random manner). For the RPs, we draw a redshift for each radio galaxy, with a fixed $\theta$ of $6 \operatorname{arcmin}$ (i.e. the mean observational separation for the RPs in the overlap region), before calculating the RM difference. To create distributions of $\triangle \mathrm{RM}$, we calculate the RM difference for 10000 draws each for both RPs and PPs. This produced smooth distributions of $\triangle \mathrm{RM}$ from which we could obtain reliable statistics.

We then generate $\triangle \mathrm{RM}$ distributions for RPs and PPs for a grid of $B_{0}$ and $l_{B}$ values. The simulations are run for a grid of $0.1 \leq$ $B_{0} \leq 10 \mathrm{nG}$ and $0.1 \leq l_{B} / \lambda_{J} \leq 10$, both with 10 even steps in log space. To extend the grid to large values of $l_{B}$, we also produce $\triangle \mathrm{RM}$ distributions for $10 \leq l_{B} / \lambda_{J} \leq 1000$ in 5 even steps in log space (i.e. for a total of 150 grid points). We employ a parallelized version of the code (using the JOBLIB PYTHON library ${ }^{4}$ ), which takes approximately 3 weeks to run on 24 cores. The median of $|\Delta R M|$ was chosen as the most robust statistic for comparison with the observational data (see Table 1$)$. This is because the mean $(\Delta \mathrm{RM})^{2}$ values from the model are strongly affected by outliers, due to the lognormal density distribution (e.g. Blasi et al. 1999).

Fig. 7 shows the value of $|\Delta R M|_{\text {median,RP }}-|\Delta R M|_{\text {median,PP }}$ for variations in $B_{0}$ and $l_{B}$. The shaded regions outline the allowed values of $B_{0}$ based on the limit of $1.9 \mathrm{rad} \mathrm{m}^{-2}$ from Section 3 . The lines show the dependence of the excess Faraday rotation contribution to RPs on $B_{0}$, for correlation lengths in the range 0.1 $\leq l_{B} / \lambda_{J} \leq 1000$. Only small variations are present in the model output for $0.1 \leq l_{B} / \lambda_{J} \leq 10$ so we just show one line for the average dependence. This places a conservative limit on the co-moving cosmological magnetic field of $B_{0}<4 \mathrm{nG}$ for correlation lengths on Mpc scales (with smaller $B_{0}$ limits for $\operatorname{larger} l_{B}$ ). This limit should not be confused with the magnetic field strength in overdensities, which can be an order of magnitude larger due to the scaling with density in this model.

\subsubsection{Limitations of the Monte Carlo model}

While the above model is a good approximation for spherical overdensities and underdense regions like voids, a major limitation of this approach is that it does not accurately describe the density variations expected in cosmic sheets and filaments. If the contribution of magnetic fields in sheets and filaments to the observed RM is significant, then it is plausible that the magnetic field limits from this model are overestimated. Further limitations of the model are the assumption of how the magnetic field scales with the electron density, as well as the existence of a single correlation scale of magnetic fields along the line of sight.

Although limited, we consider this model an advance on models with a homogenous electron density distribution and magnetic field strength along the line of sight. The inhomogeneous model can be developed further by incorporating more realistic electron density distributions that better represent cosmic sheets and filaments, in addition to better modelling of the physical properties of the sources in the sample after obtaining their redshifts.

\subsubsection{Alternative approaches to magnetic field limits}

The simplest model one can adopt is of a homogeneous universe, where the excess $\Delta \mathrm{RM}_{\mathrm{rms}}$ of $<1.9 \mathrm{rad} \mathrm{m}^{-2}$ comes from a uniform IGM along the line of sight between the RPs. In this case, we take the median redshift of the RPs to be 0.4 in order to estimate the average electron density of $n_{e}=n_{e}(0)(1+z)^{3} \sim 5 \times 10^{-7} \mathrm{~cm}^{-3}$, with an rms magnetic field in the IGM $\left(B_{\mathrm{IGM}, \mathrm{rms}}\right)$ having a coherence length $(l)$ of $1 \mathrm{Mpc}$. We take the typical distance between the RPs to be $L \sim 1 \mathrm{Gpc}$ (using the median $\Delta z$ of $\sim 0.4$ between RPs found in V19). This leads to a limit of $B_{\mathrm{IGM}, \mathrm{rms}}<260 \mathrm{nG}$, for

$$
\begin{aligned}
\sigma_{\mathrm{RM}, \mathrm{ex}}< & 1.9\left(\frac{B_{\mathrm{IGM}, \mathrm{rms}}}{260 \mathrm{nG}}\right)\left(\frac{n_{e}}{5 \times 10^{-7} \mathrm{~cm}^{-3}}\right) \\
& \times\left(\frac{l}{1 \mathrm{Mpc}} \frac{L}{1 \mathrm{Gpc}}\right)^{1 / 2} \mathrm{rad} \mathrm{m}^{-2} .
\end{aligned}
$$

However, as we expect the RM signal to be dominated by overdense regions along the line of sight (e.g. Akahori \& Ryu 2011), we consider the limits from the inhomogeneous model more appropriate, even though our inhomogeneous model does not accurately describe the filamentary structure of the cosmic web as seen in cosmological simulations.

Alternatively, one could assume the extragalactic RM variance between RPs is completely dominated by cosmic sheets and filaments (with an insignificant contribution from voids). In this case, a limit on the rms magnetic field strength in the sheets and filaments $\left(B_{\text {filament }}\right)$ can be estimated. Using the same coherence length and path-length as above, and assuming that 25 per cent of the line of sight between RPs (i.e. $f L \sim 0.25 \mathrm{Gpc}$ ) is intersected by sheets or filaments (e.g. Cautun et al. 2014), we find that an rms magnetic field strength of $\sim 26 \mathrm{nG}$ and an average electron density of $10^{-5} \mathrm{~cm}^{-3}$, could provide

$$
\begin{aligned}
\sigma_{\mathrm{RM}, \mathrm{ex}}< & 1.9\left(\frac{B_{\text {filament }}}{26 \mathrm{nG}}\right)\left(\frac{n_{e, \text { filament }}}{10^{-5} \mathrm{~cm}^{-3}}\right) \\
& \times\left(\frac{l}{1 \mathrm{Mpc}} \frac{f L}{1 \mathrm{Gpc}}\right)^{1 / 2} \mathrm{rad} \mathrm{m}^{-2} .
\end{aligned}
$$


If we further assume that the magnetic field in the filaments scales from an initial cosmological field as $\left(n_{e, \text { filament }} / n_{e}(0)\right)^{2 / 3}$, then the initial field would be $\sim 2 \mathrm{nG}$ (within a factor of 2 of our limit of $4 \mathrm{nG}$ in Section 4.1).

\subsection{Comparison with cosmological simulations}

For constraints based on a more realistic model of the universe, we use the results of recent MHD simulations considering several different scenarios for the origin and amplification of extragalactic magnetic fields (Vazza et al. 2017). The comparison that we focus on in this case is with the LOFAR RM structure function for the PPs on small angular scales (cf. Fig. 5 for $\Delta \theta<1$ arcmin). We consider this the most relevant constraint because the extragalactic contribution to the structure function may begin to dominate at small angular scales since the contribution of the Milky Way ISM is expected to decline steeply with decreasing angular scale (e.g. Akahori et al. 2013, 2014). In contrast to the model described in Section 4.1, here the three-dimensional distribution of magnetic fields and electron density in the cosmic volume are self-consistently produced by the MHD simulation, depending on different assumed scenarios for magnetogenesis. Here, we focus on three of the most realistic scenarios, within a larger survey of 25 models presented in Vazza et al. (2017). A detailed survey of all models allowed by the LOFAR data is beyond the scope of the current work, but will become more feasible when we know the redshift distribution of the LOFAR sources.

The three different scenarios were simulated in a co-moving $(85 \mathrm{Mpc})^{3}$ volume with $1024^{3}$ cells, using the ENZO code (Vazza et al. 2017). The different prescriptions for the injection and evolution of magnetic fields were (a) a primordial, uniform, volumefilling, co-moving magnetic field of $B_{0}=0.5 \mathrm{nG}$ at the beginning of the simulation; (b) a primordial model starting from the much lower level of $B_{0}=10^{-9} \mathrm{nG}$ but including a run-time modelling of dynamo amplification of the magnetic field; (c) an 'astrophysical' run in which the magnetic field is injected solely by feedback events from star-forming regions and/or active galactic nuclei. For scenario (a), a residual level of magnetization $(\sim 1-10 \mathrm{nG})$ is present everywhere in the cosmic volume. However, in scenarios (b) and (c) the average magnetization is a steeper function of density. Particularly in the astrophysical scenario, very little magnetic fields are present outside of the virial volume of matter haloes, due to the strong association between sources of magnetization and the haloes. For more details we refer the reader to Vazza et al. (2017) and Gheller \& Vazza (2019).

In order to construct synthetic RM structure functions for the PPs for each magnetogenesis scenario, simulated maps of Faraday rotation for a $4^{\circ}$ field of view were created, before obtaining deep light-cones by stacking different snapshots along the line of sight. We note that the generation of synthetic RM structure functions for RPs was beyond the scope of the current work, but will be investigated in a future publication. In detail, using different snapshots of the above runs, we integrated along light-cones up to $z=0.5$, and simulated $(\Delta \mathrm{RM})^{2}$ for PPs, by placing pairs of sources at regular intervals of $85 \mathrm{Mpc}$ (co-moving) along the line of sight (i.e. at the end of each of the co-moving volumes used to produce the stacked sequence of Faraday rotation). We first randomly drew 1500 sources, with $|\mathrm{RM}| \geq 0.03 \mathrm{rad} \mathrm{m}^{-2}$, for 22 evenly spaced redshift bins. We then computed the $\Delta \mathrm{RM}(\Delta \theta)^{2}$ statistics at each redshift, and finally produced the observable total distribution of $\Delta \operatorname{RM}(\Delta \theta)^{2}$ by weighting each structure function by the distribution function of source redshifts approximately derived from V19.

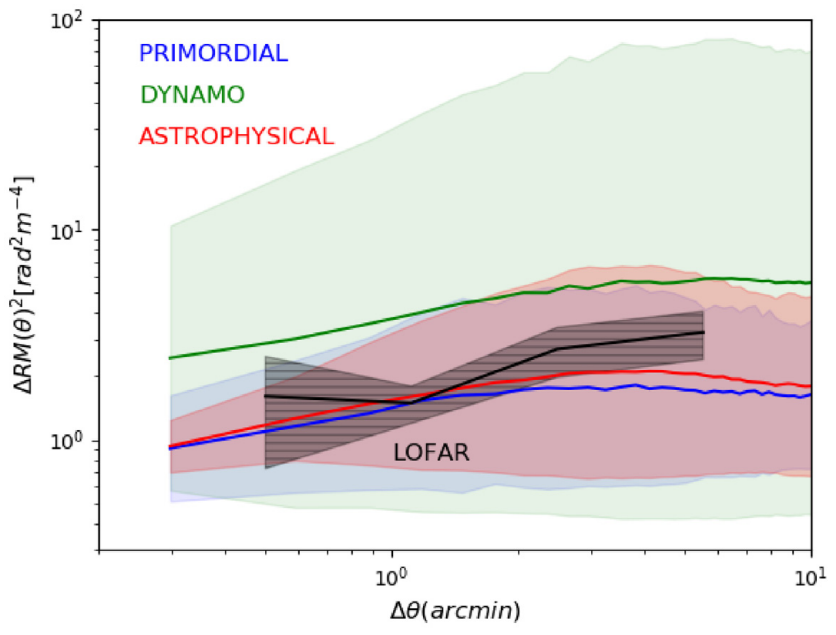

Figure 8. Simulated distribution of $\Delta \operatorname{RM}(\Delta \theta)^{2}$ as a function of angular separation for three numerical models, as described in Section 4.2, compared with LOFAR data. The solid lines show the mean values and the shaded region shows the $1 \sigma$ dispersion. The dark shaded region outlining the LOFAR data is identical to that shown in Fig. 5 for the PPs. The blue line gives the prediction for a uniform primordial model of $B_{0}=0.5 \mathrm{nG}$ (comoving). The variance around each model is due to the redshift distribution of sources.

Fig. 8 shows the simulated distribution of $\Delta \mathrm{RM}(\Delta \theta)^{2}$ as a function of angular separation for the three models, enabling a direct comparison with the LOFAR data (dark shaded region). Note that the RM variance from the Milky Way is not included in the models (so the model trends are not expected to exceed the LOFAR curve). The dynamo amplification model (green) is quite far from our LOFAR observations. Based on the typical range of magnetic field measured within filaments in these runs (Gheller \& Vazza 2019, fig. 6), this suggests a limit of $\lesssim 10$ $100 \mathrm{nG}$ on the average magnetization of filaments crossed by the polarized emission observed with LOFAR. On the other hand, the astrophysical scenario (red line) and the uniform primordial model (blue line) give a more reasonable agreement with the LOFAR data, which follows from the fact that in this case the magnetic fields in filaments are far less volume filling, with a broad distribution of values centred around $1 \mathrm{nG}$. This in turn suggests that $B_{0} \approx 0.5 \mathrm{nG}$ is the upper limit on primordial magnetic fields that can be derived from the LOFAR data. Conversely, no rescaling can reconcile the simulated statistics of $\Delta \mathrm{RM}(\Delta \theta)^{2}$ in the dynamo case, because the observed scatter in the LOFAR observations is more than one order of magnitude smaller than what is produced by the large fluctuations that are present across the distribution of filaments in the dynamo scenario (e.g. Ryu et al. 2008).

We note that the constant spatial resolution of the cosmological simulations ( $83 \mathrm{kpc}$ per cell) means that scales below 1 arcmin are not resolved by the simulation for the $z \leq 0.07$ portion of the light-cone. This likely makes our simulated distribution of $(\Delta \mathrm{RM})^{2}$ for $\Delta \theta \leq 1$ arcmin a lower limit of the true distribution that can be expected for each model. It is also important to note that the simulated pairs were not placed at the physical location of the AGN outflows. In general, this was done to avoid a strong contribution from the ambient AGN medium to all sources, which would have the effect of increasing the RM variance for a fixed primordial field strength. To remain consistent with the LOFAR data, this would then have pushed the magnetic field limits even lower and made assessing the dynamo amplification scenario in filaments 
difficult. Our approach is also potentially more consistent with the data because we do not expect to detect polarized emission from LOFAR sources embedded in dense magnetoionic media (e.g. Stuardi et al. 2020). This means the dynamo scenario is disfavoured solely in cosmic filaments, and not in denser regions like in the intracluster medium. The ability to model magnetic field fluctuations on small scales would need to be added to the simulations before a more realistic distribution of radio galaxy environments could be included. Our hypothesis for the difference between the V19 and LOFAR results (i.e. the difference being due to the ambient radio galaxy medium) could be tested with such an implementation.

\section{DISCUSSION}

The goal of this work is to isolate the extragalactic RM variance from the other contributions along the line of sight (e.g. equation 2). The RM variance introduced by the ionosphere $\left(\sigma_{\mathrm{RM}, \text { ion }}^{2}\right)$ was accounted for by restricting the RM difference of close pairs of radio sources, $(\Delta \mathrm{RM})^{2}$, to come from the same observational pointing, in addition to the standard correction of the time-variable ionosphere $\mathrm{RM}$ as described in Section 2. The RM variance contributed by measurement errors $\left(\sigma_{\mathrm{RM}, \mathrm{err}}^{2}\right)$ was subtracted from the quoted rms values, although the effect of this is minor due to the small LOFAR RM errors $\left(\sim 0.03 \mathrm{rad} \mathrm{m}^{-2}\right)$. We then split the $(\Delta \mathrm{RM})^{2}$ sample into pairs from the same physical source (PPs) and non-physical, random pairs on the sky (RPs). The comparison of these two samples can then be used to account for the Milky Way contribution $\left(\sigma_{\mathrm{RM}, \mathrm{MW}}^{2}\right)$ in a statistical sense. In principle, this leaves only the contribution from extragalactic Faraday rotation $\left(\sigma_{\mathrm{RM}, \mathrm{ex}}^{2}\right)$. By analysing the difference in $(\Delta \mathrm{RM})^{2}$ between RPs and PPs in Section 3, we limit the extragalactic RM contribution between the LOFAR RPs to $<1.9 \mathrm{rad} \mathrm{m}^{-2}$ ( $\sim 95$ per cent confidence).

\subsection{The Faraday medium local to radio sources}

For the discussion, we split the extragalactic RM variance into contributions local to the source $\left(\sigma_{\mathrm{RM}, \text { local }}^{2}\right)$ and from the intergalactic medium in the more distant foreground $\left(\sigma_{\mathrm{RM} \text {,IGMF }}^{2}\right)$. At $1.4 \mathrm{GHz}$, V19 found an rms difference of $\sim 5-10 \mathrm{rad} \mathrm{m}^{-2}$ between RPs and PPs. This is similar to $\sigma_{\mathrm{RM} \text {,ex }} \sim 7 \mathrm{rad} \mathrm{m}^{-2}$ estimated by Schnitzeler (2010) and Oppermann et al. (2015) at $1.4 \mathrm{GHz}$. However, our result at $144 \mathrm{MHz}$ (i.e. $\sigma_{\mathrm{RM} \text {,ex }}<1.9 \mathrm{rad} \mathrm{m}^{-2}$ ) appears to be in conflict with the $1.4 \mathrm{GHz}$ results, as one does not expect a strong frequency-

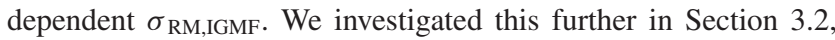
where we found that (a) the majority of the $144 \mathrm{MHz}$ polarized sources were not detected in the NVSS at $1.4 \mathrm{GHz}$ (because they are too faint, as LoTSS is $\sim 10$ times more sensitive for steep spectrum sources), (b) most polarized sources at $1.4 \mathrm{GHz}$ are not detected at $144 \mathrm{MHz}$ (due to Faraday depolarization), and importantly (c) the polarized sources in common have a smaller $\Delta \mathrm{RM}_{\mathrm{rms}}$ (and degree of polarization) at $144 \mathrm{MHz}$. This indicates that the larger rms difference of $\sim 5-10 \mathrm{rad} \mathrm{m}^{-2}$ found in V19 between RPs and PPs is due to RM variance in the magnetized environment local to the sources (i.e. from $\sigma_{\mathrm{RM}, \text { local }}^{2}$ ).

For example, for a polarized signal to be detected at $144 \mathrm{MHz}$, only small amounts of Faraday depolarization within the LOFAR synthesized beam are possible (e.g. less than $0.4 \mathrm{rad} \mathrm{m}^{-2}$ on scales $<20$ arcsec for the most common model of external Faraday dispersion, Burn (1966), with $\left.p(\lambda) \propto \mathrm{e}^{-2 \sigma_{\mathrm{RM}}^{2} \lambda^{4}}\right)$. Alternatively, the polarized signal may originate from a compact emission region on sub-beam scales (e.g. hotspots), and thus the inferred Faraday depolarization would not represent the RM variance on larger scales (i.e. as would be relevant for physical pairs on scales $>100 \mathrm{kpc}$ ).

In any case, for radio sources in regions of dense magnetized gas, such as near the centre of groups and clusters of galaxies, there is likely too much Faraday depolarization for emission to be detected at $144 \mathrm{MHz}$. Furthermore, large asymmetries in the Faraday rotation properties of opposite lobes are often found in these rich environments due to, for example, the Laing-Garrington effect (Garrington et al. 1988; Laing 1988), which would make the detection of polarized emission from physical pairs less likely compared to physically large radio sources that are closer to the plane of the sky (e.g. Saripalli \& Subrahmanyan 2009). This is consistent with recent results that find the majority of polarized detections in LOFAR data are from hotspots of FRII radio galaxies that are not associated with galaxy clusters and have large physical sizes (O'Sullivan et al. 2018a; Stuardi et al. 2020; Mahatma et al. in preparation). Therefore, we expect that LOFAR polarized sources are typically located in regions of the Universe with low RM variance. This makes them ideal probes of the weak magnetization of the cosmic filaments and voids far from galaxy cluster environments.

\subsection{Model limits on intergalactic magnetic fields}

In Section 4, we take two approaches to deriving upper limits on the co-moving cosmological magnetic field strength. In one approach, we use a Monte Carlo model to generate distributions of $\triangle \mathrm{RM}$ for RPs and PPs in a universe with an inhomogeneous matter distribution and with a magnetic field strength that scales with the density inhomogeneities (i.e. $B \propto n_{e}^{2 / 3}$ ). The model allows us to explore a wide range of input co-moving cosmological magnetic field strengths $\left(0.1 \leq B_{0} \leq 10 \mathrm{nG}\right)$ and correlations lengths $\left(0.1 \leq l_{B} / \lambda_{J} \leq 1000\right)$. For this model, we find that the median $|\triangle R M|$ is the best statistical indicator due to the highly nonGaussian $\triangle \mathrm{RM}$ distribution. Using the observational constraint of the difference in the median $|\triangle \mathrm{RM}|$ between RPs and PPs being $<1.9 \mathrm{rad} \mathrm{m}^{-2}$ provides an upper limit of $B_{0}<4 \mathrm{nG}$ for magnetic field correlation lengths in the range $0.1 \leq l_{B} / \lambda_{J} \leq 10$ (where $\lambda_{J} \sim$ $2.3 \mathrm{Mpc}$ at $z=0$ ). This limit is comparable with upper limits on the primordial field from CMB measurements (Planck Collaboration XIX 2016b), and almost 10 times lower than the upper limit of $\sim 37 \mathrm{nG}$ derived in V19.

In the second approach, we compare our observational results with cosmological MHD simulations (Vazza et al. 2017) in three different scenarios: (a) a strong initial primordial field of $B_{0}=0.5 \mathrm{nG}$, (b) a primordial field of $B_{0}=10^{-9} \mathrm{nG}$ with dynamo amplification, and (c) magnetization only from AGN and galactic outflows. In this approach, the most useful constraint comes from the RM structure function on the smallest angular scales because this should have the smallest contribution from the RM variance of the Milky Way (which is not included in the models). In particular, synthetic RM structure functions for PPs were created from the simulations (as described in Section 4.2) and constrained by the data for angular separations less than $1 \operatorname{arcmin}$ (i.e. $\left.\left\langle(\Delta \mathrm{RM})^{2}\right\rangle<1.6 \mathrm{rad}^{2} \mathrm{~m}^{-4}\right)$. Both the scenario of magnetization by astrophysical processes (e.g. AGN and galactic outflows) and the primordial case are consistent with the data, for an initial (spatially uniform) primordial seed field of $B_{0}$ $\lesssim 0.5 \mathrm{nG}$. The dynamo amplification scenario is inconsistent with the data as it produces $\Delta \mathrm{RM}$ fluctuations that are too large.

These inferences can be considered preliminary, since one of the main limitations of the comparison between the models and the data is our lack of knowledge of the exact redshift distribution 
of the observed radio sources. For example, we do not know the true distribution of physical (and angular) separations for PPs as a function of redshift. Also, the simulated PPs are not placed at the location of AGN outflows (i.e. no model contribution of $\sigma_{\mathrm{RM}, \text { local }}^{2}$ ), which leads to more conservative upper limits on the seed field and provides constraints that are more relevant to the dynamo amplification of field in filaments (rather than in more dense regions such as near galaxy clusters). Furthermore, we have not included a model for the RPs, mainly due to the lack of redshift information. For future work, in addition to more realistic models based on observed redshifts and environments, we plan to explore how the LOFAR data might also constrain the morphology of primordial magnetic fields, whose initial spectra are already constrained by PLANCK observations (Planck Collaboration XIX 2016b). In general, this highlights the potential of LOFAR data to realistically discriminate between competing magnetogenesis scenarios.

\subsection{Upcoming advances}

Much more can be achieved in the near future with LOFAR. In particular, we expect the sample of pairs to potentially increase by an order of magnitude for the full LoTSS survey, helping to push well into the sub-nG regime for the study of cosmic magnetic fields. In the near term, host galaxy identifications and redshifts will be provided by the value-added data products in LoTSS DR2. We expect to get photometric or spectroscopic redshifts for $\sim 80$ per cent of the polarized sources in our current sample (O'Sullivan et al. 2018a). With the LOFAR-WEAVE survey (Smith et al. 2016), we expect spectroscopic redshifts for all the polarized radio sources in LoTSS up to at least $z=1$. In combination with the high-fidelity 6 arcsec total intensity images provided by the LoTSS survey, these redshifts will enable precise linear size estimates of the sources, which will further enhance our ability to distinguish between magnetoionic material local to the source and that associated with cosmic filaments and voids. In addition, splitting the sample into redshift bins (in addition to $\Delta z$ bins for the random pairs) will allow investigations of the evolution of magnetic fields with cosmic time.

In order to learn more about the properties of LOFAR polarized sources (and the IGMF), we will need to consider several other properties, such as the degree of polarization/depolarization, the total intensity spectral index, the radio source morphology, the environment, etc. Such investigations are important to allow a better understanding of the different astrophysical contributions to the total observed RM variance, to weight the RM variance of each sub-population in an appropriate manner (e.g. Rudnick 2019), and to potentially remove blazars from the sample. This should be done in combination with other upcoming RM surveys at higher frequencies (e.g. POSSUM, VLASS; Gaensler et al. 2010; Lacy et al. 2020), which can probe cosmic magnetic fields in highdensity environments that are currently inaccessible for LOFAR. In the longer term, both the SKA-Low and SKA-Mid (e.g. Braun et al. 2015 , and references therein) will be essential to further map out the frequency-dependent behaviour of the extragalactic RM variance in order to uncover the nature of magnetic fields in the cosmic web.

\section{CONCLUSIONS}

We have presented a Faraday rotation study of 349 close pairs of extragalactic radio sources with LOFAR, to investigate the properties of extragalactic magnetic fields. The data used are from the ongoing LoTSS (Shimwell et al. 2019), which is imaging the northern sky in continuum polarization from 120 to $168 \mathrm{MHz}$. The large bandwidth at such low frequencies provides exceptional RM precision, with typical errors of $\sim 0.03 \mathrm{rad} \mathrm{m}^{-2}$, which are $\sim 300$ times better than available for previous studies (e.g. Vernstrom et al. 2019).

By considering the variance of the RM difference between physical pairs (e.g. double-lobed radio galaxies) and non-physical, random pairs (i.e. physically different sources with close projected separations on the sky), we statistically separate the extragalactic component of the RM variance from that due to the Milky Way. In the region of overlapping angular scales from 2 to 10 arcmin, we find a trimmed rms RM difference of $1.8 \pm 0.3 \mathrm{rad} \mathrm{m}^{-2}$ for 41 random pairs and $1.4 \pm 0.2 \mathrm{rad} \mathrm{m}^{-2}$ for 75 physical pairs, providing an estimate of $+0.4 \pm 0.3 \mathrm{rad} \mathrm{m}^{-2}$ for the excess Faraday rotation experienced by random pairs. A similar estimate of $+0.3 \pm 0.8 \mathrm{rad} \mathrm{m}^{-2}$ is found from an analysis of the median $|\Delta \mathrm{RM}|$. A KS test indicates that there is no significant difference between the $\Delta \mathrm{RM}$ distributions of random and physical pairs in the region of overlapping angular scales. Using the difference in the median $|\triangle R M|$ values, we place an upper limit of $1.9 \mathrm{rad} \mathrm{m}^{-2}$ ( $\sim 95$ per cent confidence) on the excess extragalactic Faraday rotation contribution to random pairs over physical pairs.

This result is in apparent conflict with estimates of the extragalactic variance of $\sim 5-10 \mathrm{rad} \mathrm{m}^{-2}$ derived from observations at $1.4 \mathrm{GHz}$ (Vernstrom et al. 2019). There is no expectation of a frequencydependent RM from magnetic fields in cosmic filaments and voids. Therefore, our results point to the contribution of magnetoionic material local to the radio source as the dominant extragalactic contribution at $1.4 \mathrm{GHz}$ (e.g. the magnetized IGM of galaxy groups and clusters). This means that sources in Vernstrom et al. (2019) with large RM variance local to the source are depolarized below the detection limit at $144 \mathrm{MHz}$. With these sources missing from the LOFAR sample, our data are probing the low RM variance Universe, providing even more stringent constraints on the magnetization of the cosmic web away from galaxy cluster environments.

To investigate the implication of our results for the strength of the co-moving cosmological magnetic field $\left(B_{0}\right)$, we use a model of an inhomogeneous universe to calculate the RM difference between adjacent pairs of cosmic sightlines. We use this model to generate $\triangle \mathrm{RM}$ distributions for random and physical pairs for a wide range of input values of $B_{0}$ and the field correlation length. This allows us to place a limit of $B_{0}<4 \mathrm{nG}$ on Mpc scales.

We also compare our results with a suite of cosmological MHD simulations, allowing us to investigate different magnetogenesis scenarios. In particular, we investigated the RM variance generated in three different scenarios: a strong initial primordial field of $0.5 \mathrm{nG}$, a weak primordial field of $10^{-9} \mathrm{nG}$ but with dynamo amplification, and an astrophysical scenario where magnetic field is injected solely by AGN and galactic outflows. To constrain the different simulation scenarios, we use the observed RM structure function of physical pairs on angular scales less than 1 arcmin, because these data should have the lowest RM variance contribution from the Milky Way (which is not included in the model). We find that both the astrophysical scenario and a primordial scenario (with a seed field of $B \lesssim 0.5 \mathrm{nG}$ ) are consistent with the current data. Interestingly, the dynamo amplification in cosmic filaments is disfavoured because the RM dispersion is much larger than the observed scatter in the LOFAR data.

In the coming years, we will be able to significantly expand on the current sample, in addition to adding redshift information for the host galaxies of the radio sources. This will allow us to push into the sub-nG regime and further constrain both the origin and evolution of cosmic magnetic fields on large scales. 


\section{ACKNOWLEDGEMENTS}

SPO and MB acknowledge financial support from the Deutsche Forschungsgemeinschaft (DFG) under grant BR2026/23. MB acknowledges support from the Deutsche Forschungsgemeinschaft under Germany's Excellence Strategy - EXC 2121 'Quantum Universe' - 390833306. FV and NTL acknowledge financial support from the Horizon 2020 programme under the ERC Starting Grant 'MAGCOW', no. 714196. The ENZO (enzo-project.org) simulations used for this work were produced on the CSCS Supercomputer of ETHZ (Lugano) and on the Marconi Supercomputer at CINECA (Bologna), under project no. INA17_C4A28 with FV as PI. FV gratefully acknowledge the usage of online storage tools kindly provided by the INAF Astronomical Archive (IA2) initiative (http: //www.ia2.inaf.it). CS acknowledges support from the ERC-StG DRANOEL, no. 714245. LOFAR (van Haarlem et al. 2013) is the Low Frequency Array designed and constructed by ASTRON. It has observing, data processing, and data storage facilities in several countries that are owned by various parties (each with their own funding sources), and that are collectively operated by the ILT foundation under a joint scientific policy. The ILT resources have benefitted from the following recent major funding sources: CNRSINSU, Observatoire de Paris and Université d'Orléans, France; BMBF, MIWF-NRW, MPG, Germany; Science Foundation Ireland (SFI), Department of Business, Enterprise and Innovation (DBEI), Ireland; NWO, The Netherlands; The Science and Technology Facilities Council, UK; Ministry of Science and Higher Education, Poland. Part of this work was carried out on the Dutch national e-infrastructure with the support of the SURF Cooperative through grant e-infra 160022 and 160152. The LOFAR software and dedicated reduction packages on https://github.com/apmechev/GR ID_LRT were deployed on the e-infrastructure by the LOFAR e-infragroup, consisting of J. B. R. Oonk (ASTRON \& Leiden Observatory), A. P. Mechev (Leiden Observatory), and T. Shimwell (ASTRON) with support from N. Danezi (SURFsara) and C. Schrijvers (SURFsara). This research has made use of data analysed using the University of Hertfordshire high-performance computing facility (http://uhhpc.herts.ac.uk/) and the LOFAR-UK computing facility located at the University of Hertfordshire and supported by STFC [ST/P000096/1]. SPO thanks Marcel van Daalen for feedback on some aspects of the Monte Carlo model for the RM pairs. This research made use of ASTROPY, a community-developed core PYTHON package for astronomy (Astropy Collaboration 2013) hosted at http://www.astropy.org/, MATPLOTLIB (Hunter 2007), APLPY (Robitaille \& Bressert 2012), an open-source astronomical plotting package for PYTHON hosted at http://aplpy.github.com/, and TOPCAT, an interactive graphical viewer and editor for tabular data (Taylor 2005). The authors thank the referee, Prof. Lawrence Rudnick, for a helpful review.

\section{REFERENCES}

Akahori T., Ryu D., 2011, ApJ, 738, 134

Akahori T., Ryu D., Kim J., Gaensler B. M., 2013, ApJ, 767, 150

Akahori T., Gaensler B. M., Ryu D., 2014, ApJ, 790, 123

Akahori T. et al., 2018, PASJ, 70, R2

Anderson C. S., Gaensler B. M., Heald G. H., O'Sullivan S. P., Kaczmarek J. F., Feain I. J., 2018, ApJ, 855, 41

Astropy Collaboration, 2013, A\&A, 558, A33

Banfield J. K., O’Sullivan S. P., Wieringa M. H., Emonts B. H. C., 2019, MNRAS, 482, 5250

Baratta P., Bel J., Plaszczynski S., Ealet A., 2019, A\&A, 633, A26
Beck R., 2015, in Lazarian A., de Gouveia Dal Pino E. M., Melioli C., eds, Astrophysics and Space Science Library, Vol. 407, Magnetic Fields in Diffuse Media. Springer, Berlin, p. 507

Beck A. M., Hanasz M., Lesch H., Remus R.-S., Stasyszyn F. A., 2013, MNRAS, 429, L60

Bi H., Davidsen A. F., 1997, ApJ, 479, 523

Blasi P., Burles S., Olinto A. V., 1999, ApJ, 514, L79

Botteon A. et al., 2018, MNRAS, 478, 885

Braun R., Bourke T., Green J. A., Keane E., Wagg J., 2015, in Proc. Sci., Advancing Astrophysics with the Square Kilometre Array (AASKA14). SISSA, Trieste, PoS\#174

Bray J. D., Scaife A. M. M., 2018, ApJ, 861, 3

Brentjens M. A., de Bruyn A. G., 2005, A\&A, 441, 1217

Brown S. et al., 2017, MNRAS, 468, 4246

Burn B. J., 1966, MNRAS, 133, 67

Carilli C. L., Taylor G. B., 2002, ARA\&A, 40, 319

Cautun M., van de Weygaert R., Jones B. J. T., Frenk C. S., 2014, MNRAS, 441,2923

Chuang C.-H. et al., 2015, MNRAS, 452, 686

Coles P., Jones B., 1991, MNRAS, 248, 1

Condon J. J., Cotton W. D., Greisen E. W., Yin Q. F., Perley R. A., Taylor G. B., Broderick J. J., 1998, AJ, 115, 1693

Csáki C., Kaloper N., Peloso M., Terning J., 2003, J. Cosmol. Astropart. Phys., 2003, 005

de Gasperin F., Mevius M., Rafferty D. A., Intema H. T., Fallows R. A., 2018, A\&A, 615, A179

de Gasperin F. et al., 2019, A\&A, 622, A5

Dermer C. D., Cavadini M., Razzaque S., Finke J. D., Chiang J., Lott B., 2011, ApJ, 733, L21

Dolag K., Kachelriess M., Ostapchenko S., Tomàs R., 2011, ApJ, 727, L4

Donnert J., Dolag K., Lesch H., Müller E., 2009, MNRAS, 392, 1008

Durrer R., Neronov A., 2013, A\&AR, 21, 62

Farnsworth D., Rudnick L., Brown S., 2011, AJ, 141, 191

Furlanetto S. R., Loeb A., 2001, ApJ, 556, 619

Gaensler B. M., Beck R., Feretti L., 2004, New Astron. Rev., 48, 1003

Gaensler B. M., Landecker T. L., Taylor A. R., POSSUM Collaboration, 2010, in AAS Meeting Abstract, \#470.13

Garrington S. T., Leahy J. P., Conway R. G., Laing R. A., 1988, Nature, 331,147

George S. J., Stil J. M., Keller B. W., 2012, PASA, 29, 214

Gheller C., Vazza F., 2019, MNRAS, 486, 981

Govoni F. et al., 2019, Science, 364, 981

Grasso D., Rubinstein H. R., 2001, Phys. Rep., 348, 163

Hackstein S., Vazza F., Brüggen M., Sigl G., Dundovic A., 2016, MNRAS, 462, 3660

Hackstein S., Brüggen M., Vazza F., Gaensler B. M., Heesen V., 2019, MNRAS, 488, 4220

Hardcastle M. J. et al., 2019, A\&A, 622, A12

Harvey-Smith L., Madsen G. J., Gaensler B. M., 2011, ApJ, 736, 83

Horns D., Maccione L., Meyer M., Mirizzi A., Montanino D., Roncadelli M., 2012, Phys. Rev. D, 86, 075024

Hunter J. D., 2007, Comput. Sci. Eng., 9, 90

Hutschenreuter S., Enßlin T. A., 2019, A\&A, 633, A150

Jedamzik K., Pogosian L., 2020, preprint (arXiv:2004.09487)

Knuettel S., O’Sullivan S. P., Curiel S., Emonts B. H. C., 2019, MNRAS, 482, 4606

Kolatt T., 1998, ApJ, 495, 564

Kronberg P. P., Perry J. J., 1982, ApJ, 263, 518

Kulsrud R. M., Zweibel E. G., 2008, Rep. Prog. Phys., 71, 046901

Lacy M. et al., 2020, PASP, 132, 035001

Laing R. A., 1988, Nature, 331, 149

Laing R. A., Bridle A. H., Parma P., Murgia M., 2008, MNRAS, 391, 521

Locatelli N., Vazza F., Domínguez-Fernández P., 2018, Galaxies, 6, 128

Mo H., van den Bosch F. C., White S., 2010, Galaxy Formation and Evolution. Cambridge Univ. Press, Cambridge 
Montanino D., Vazza F., Mirizzi A., Viel M., 2017, Phys. Rev. Lett., 119, 101101

Neld A. et al., 2018, A\&A, 617, A136

Neronov A., Vovk I., 2010, Science, 328, 73

O'Sullivan S. P. et al., 2013, ApJ, 764, 162

O’Sullivan S. P. et al., 2018a, Galaxies, 6, 126

O'Sullivan S. P., Lenc E., Anderson C. S., Gaensler B. M., Murphy T., 2018b, MNRAS, 475, 4263

Oppermann N. et al., 2012, A\&A, 542, A93

Oppermann N. et al., 2015, A\&A, 575, A118

Oren A. L., Wolfe A. M., 1995, ApJ, 445, 624

Planck Collaboration XIII 2016a, A\&A, 594, A13

Planck Collaboration XIX, 2016b, A\&A, 594, A19

Pshirkov M. S., Tinyakov P. G., Urban F. R., 2016, Phys. Rev. Lett., 116, 191302

Purcell C. R. et al., 2015, ApJ, 804, 22

Raffelt G., Stodolsky L., 1988, Phys. Rev. D, 37, 1237

Ravi V. et al., 2016, Science, 354, 1249

Robitaille T., Bressert E., 2012, Astrophysics Source Code Library, record ascl:1208.017

Rudnick L., 2019, preprint (arXiv:1901.09074)

Rudnick L., Blundell K. M., 2003, ApJ, 588, 143

Ryu D., Kang H., Cho J., Das S., 2008, Science, 320, 909

Saripalli L., Subrahmanyan R., 2009, ApJ, 695, 156

Schnitzeler D. H. F. M., 2010, MNRAS, 409, L99

Shimwell T. W. et al., 2019, A\&A, 622, A1

Smith D. J. B. et al., 2016, in Reylé C., Richard J., Cambrésy L., Deleuil M., Pécontal E., Tresse L., Vauglin I., eds, SF2A-2016: Proceedings of the Annual meeting of the French Society of Astronomy and Astrophysics, Societe Francaise d'Astronomie et d'Astrophysique, p. 271

Sotomayor-Beltran C. et al., 2013, A\&A, 552, A58

Stasyszyn F., Nuza S. E., Dolag K., Beck R., Donnert J., 2010, MNRAS, 408,684

Stil J. M., Taylor A. R., Sunstrum C., 2011, ApJ, 726, 4

Stuardi C. et al., 2020, A\&A, preprint (arXiv:2004.05169)

Subramanian K., 2016, Rep. Prog. Phys., 79, 076901

Tavecchio F., Ghisellini G., Bonnoli G., Foschini L., 2011, MNRAS, 414, 3566
Taylor M. B., 2005, in Shopbell P., Britton M., Ebert R., eds, ASP Conf. Ser. Vol. 347, Astronomical Data Analysis Software and Systems XIV. Astron. Soc. Pac., San Francisco, p. 29

Taylor A. R., Stil J. M., Sunstrum C., 2009, ApJ, 702, 1230

Taylor A. M., Vovk I., Neronov A., 2011, A\&A, 529, A144

Vacca V. et al., 2018, MNRAS, 479, 776

Van Eck C. L. et al., 2018, A\&A, 613, A58

van Haarlem M. P. et al., 2013, A\&A, 556, A2

van Weeren R. J., de Gasperin F., Akamatsu H., Brüggen M., Feretti L., Kang H., Stroe A., Zandanel F., 2019, Space Sci. Rev., 215, 16

Vazza F., Brüggen M., Gheller C., Hackstein S., Wittor D., Hinz P. M., 2017, Class. Quantum Gravity, 34, 234001

Vazza F., Ettori S., Roncarelli M., Angelinelli M., Brüggen M., Gheller C., 2019, A\&A, 627, A5

Vernstrom T., Gaensler B. M., Brown S., Lenc E., Norris R. P., 2017, MNRAS, 467, 4914

Vernstrom T., Gaensler B. M., Rudnick L., Andernach H., 2019, ApJ, 878, 92( V19)

Widrow L. M., 2002, Rev. Mod. Phys., 74, 775

Widrow L. M., Ryu D., Schleicher D. R. G., Subramanian K., Tsagas C. G., Treumann R. A., 2012, Space Sci. Rev., 166, 37

Zweibel E. G., Heiles C., 1997, Nature, 385, 131

\section{SUPPORTING INFORMATION}

Supplementary data are available at MNRAS online.

\section{RMpairs_Table_2.dat}

Please note: Oxford University Press is not responsible for the content or functionality of any supporting materials supplied by the authors. Any queries (other than missing material) should be directed to the corresponding author for the article.

This paper has been typeset from a $\mathrm{T}_{\mathrm{E}} \mathrm{X} / \mathrm{L} \mathrm{T} \mathrm{E} \mathrm{X}$ file prepared by the author. 